\title{
Fully-Abstract Compilation by Approximate Back-Translation
}

\author{
Dominique Devriese Marco Patrignani * Frank Piessens \\ iMinds-Distrinet, KU Leuven, Belgium \\ first.last @ cs.kuleuven.be
}

\begin{abstract}
A compiler is fully-abstract if the compilation from source language programs to target language programs reflects and preserves behavioural equivalence. Such compilers have important security benefits, as they limit the power of an attacker interacting with the program in the target language to that of an attacker interacting with the program in the source language. Proving compiler fullabstraction is, however, rather complicated. A common proof technique is based on the back-translation of target-level program contexts to behaviourally-equivalent source-level contexts. However, constructing such a back-translation is problematic when the source language is not strong enough to embed an encoding of the target language. For instance, when compiling from the simply-typed $\lambda$ calculus $\left(\lambda^{\tau}\right)$ to the untyped $\lambda$-calculus $\left(\lambda^{\mathrm{u}}\right)$, the lack of recursive types in $\lambda^{\tau}$ prevents such a back-translation.

We propose a general and elegant solution for this problem. The key insight is that it suffices to construct an approximate backtranslation. The approximation is only accurate up to a certain number of steps and conservative beyond that, in the sense that the context generated by the back-translation may diverge when the original would not, but not vice versa. Based on this insight, we describe a general technique for proving compiler full-abstraction and demonstrate it on a compiler from $\lambda^{\tau}$ to $\lambda^{\mathrm{u}}$. The proof uses asymmetric cross-language logical relations and makes innovative use of step-indexing to express the relation between a context and its approximate back-translation. We believe this proof technique can scale to challenging settings and enable simpler, more scalable proofs of compiler full-abstraction.
\end{abstract}

Categories and Subject Descriptors D.2.4 [Software/Program Verification]: Correctness Proofs; D.3.4 [Processors]: Compilers; F.3.2 [Semantics of Programming Languages]: Operational Semantics

Keywords Fully-abstract compilation, logical relations, crosslanguage logical relations, step-indexed logical relations, compiler security, secure compilation

We typeset source and target language terms in green resp. pink; we recommend to print this paper in colour for maximum clarity.

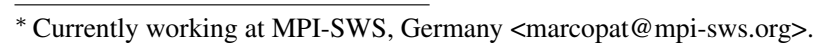

Copyright (c) ACM, 2016. This is the author's version of the work. It is posted here by permission of ACM for your personal use. Not for redistribution. The definitive version was published in POPL '16, January 20-22, 2016, St. Petersburg, FL, USA, http://doi.acm.org/10.1145/10.1145/2837614.2837618.

Copyright (c) ACM 978-1-4503-3549-2/16/01_..\$15.00

\section{Introduction}

A compiler is fully-abstract if the compilation from source language programs to target language programs preserves and reflects behavioural equivalence [Abadi, 1999, Gorla and Nestman, 2014]. Such compilers have important security benefits. It is often realistic to assume that attackers can interact with a program in the target language, and depending on the target language this can enable attacks such as improper stack manipulation, breaking control flow guarantees, reading from or writing to private memory of other components, inspecting or modifying the implementation of a function etc. [Abadi, 1999, Kennedy, 2006, Patrignani et al., 2015, Abadi and Plotkin, 2012, Fournet et al., 2013, Agten et al., 2012]. A fully-abstract compiler is sufficiently defensive to rule out such attacks: the power of an attacker interacting with the program in the target language is limited to attacks that could also be performed by an attacker interacting with the program in the source language.

Formally, we model a compiler as a function $\llbracket \cdot \rrbracket$ that maps source language terms $t$ to target language terms $\llbracket t \rrbracket$. Elements of the source language are typeset in a green, bold font, while elements of the target language are typeset in a pink, sans-serif font. Roughly, the compiler is fully-abstract, if for any two source language terms $t_{1}$ and $t_{2}$, we have that they are behaviourally equivalent $\left(t_{1} \simeq_{c t x} t_{2}\right)$ if and only if their compiled counterparts are behaviourally equivalent $\left(\llbracket \mathrm{t}_{1} \rrbracket \simeq{ }_{c t x} \llbracket \mathrm{t}_{2} \rrbracket\right)$ [Abadi, 1999]. The notion of behavioural equivalence used here is the canonical notion of contextual equivalence: two terms are equivalent if they behave the same when plugged into any valid context. Specifically, we take contextual equivalence to be equi-termination: $t \simeq_{c t x} t^{\prime} \stackrel{\text { def }}{=} \forall \mathfrak{C}, \mathfrak{C}[t] \Downarrow \Longleftrightarrow \mathfrak{C}\left[t^{\prime}\right] \Downarrow$. The universal quantification over contexts $\mathfrak{C}$ ensures that the results produced by $t$ and $t^{\prime}$ are the same [Plotkin, 1977, Curien, 2007].

The full-abstraction property can be split into two parts: the right-to-left implication and the left-to-right implication, which we call (contextual) equivalence reflection and preservation respectively.

Equivalence reflection $\left(\mathrm{t}_{1} \simeq_{c t x} \mathrm{t}_{2} \Leftarrow \llbracket \mathrm{t}_{1} \rrbracket \simeq_{c t x} \llbracket \mathrm{t}_{2} \rrbracket\right)$ requires that if the compiler produces equivalent target programs, then the source programs must have been equivalent. In other words, nonequivalent source programs must be compiled to non-equivalent target programs. Intuitively, this property captures an aspect of compiler correctness: if programs with different source language behaviour become equivalent after compilation, the compiler must have incorrectly compiled at least one of them.

We build on cross-language logical relations: a technique that has recently been proposed for proving compiler correctness [Hur and Dreyer, 2011, Benton and Hur, 2009, 2010]. The general idea of this approach is depicted in Fig. 1 (purposely ignoring languagespecific things such as the types of the terms involved). The proof starts from the knowledge that $\llbracket \mathrm{t}_{1} \rrbracket \simeq_{c t x} \llbracket \mathrm{t}_{2} \rrbracket$ and needs to prove that $\mathrm{t}_{1} \simeq_{c t x} \mathrm{t}_{2}$. That is, for an arbitrary valid context $\mathfrak{C}$, it shows 


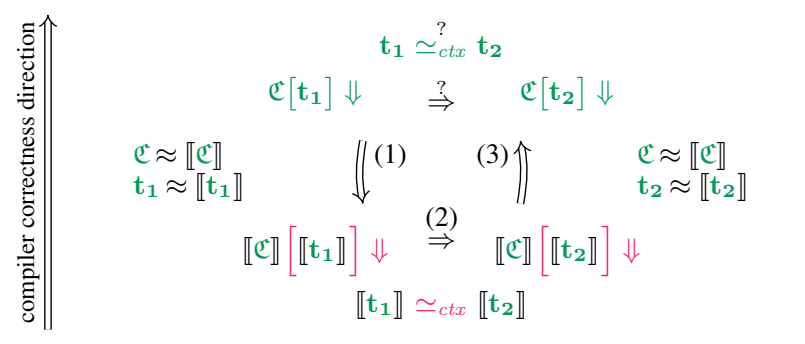

Figure 1. Proving one half of full-abstraction: compiler correctness. Only one direction of this half is presented $(\Rightarrow)$, the other one follows by symmetry.

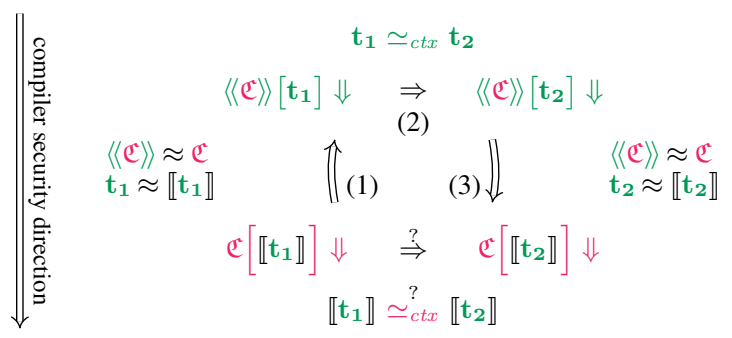

Figure 2. Proving the other half of full-abstraction: compiler security.

that $\mathfrak{C}\left[\mathrm{t}_{1}\right] \Downarrow$ if and only if $\mathfrak{C}^{\mathfrak{C}}\left[\mathrm{t}_{2}\right] \Downarrow$. By symmetry, it suffices to show that $\mathfrak{C}\left[\mathbf{t}_{1}\right] \Downarrow \Rightarrow \mathfrak{C}\left[\mathrm{t}_{2}\right] \Downarrow$.

The idea of the approach is to define a cross-language logical relation $\mathrm{t} \approx \mathrm{t}$ that expresses when a compiled term $\mathrm{t}$ behaves as a target-level version of source-level term $t$. This logical relation is not compiler-specific: it should be understood as a specification of a target-level calling convention rather than precise representation choices for a specific compiler. If we can then prove that any term is logically related to its compilation $(\mathrm{t} \approx \llbracket \mathrm{t} \rrbracket)$, and that the same result holds for contexts $(\mathfrak{C} \approx \llbracket \mathfrak{C} \rrbracket)$, then equivalence reflection follows. Starting from $\mathrm{t}_{1} \approx \llbracket \mathrm{t}_{1} \rrbracket$ and $\mathrm{t}_{2} \approx \llbracket \mathrm{t}_{2} \rrbracket$ and $\mathfrak{C} \approx \llbracket \mathfrak{C} \rrbracket$, the proof uses the inherent compositionality of logical relations to know $\mathfrak{C}\left[\mathrm{t}_{1}\right] \approx \llbracket \mathfrak{C} \rrbracket\left[\llbracket \mathrm{t}_{1} \rrbracket\right]$ and the same for $\mathrm{t}_{2}$. If the logical relations are constructed adequately, then related terms will necessarily equiterminate. Thus, $\mathfrak{C}\left[\mathbf{t}_{1}\right] \Downarrow$ iff $\llbracket \mathfrak{C} \rrbracket\left[\llbracket \mathrm{t}_{1} \rrbracket\right] \Downarrow$ and similarly for $\mathbf{t}_{2}$. In particular, this yields the implications (1) and (3) in Fig. 1. Since implication (2) follows directly from the hypothesis of (contextual) equivalence for $\llbracket t_{1} \rrbracket$ and $\llbracket t_{2} \rrbracket$, the proof for equivalence reflection is finished.

Equivalence preservation $\left(\mathrm{t}_{1} \simeq_{c t x} \mathrm{t}_{2} \Rightarrow \llbracket \mathrm{t}_{1} \rrbracket \simeq_{c t x} \llbracket \mathrm{t}_{2} \rrbracket\right)$ requires that equivalent programs remain equivalent after compilation. This means that no matter what target-level manipulations are done on compiled programs, the programs must behave equivalently if the source programs were equivalent. This precludes all sorts of target-level attacks that break source-level guarantees.

If the source language is strong enough, it is possible to apply a strategy analogous to proving equivalence reflection for proving preservation, as depicted in Fig. 2. ${ }^{1}$ Given an arbitrary target-level context $\mathfrak{C}$, we need to prove that $\mathfrak{C}\left[\llbracket \mathrm{t}_{1} \rrbracket\right] \Downarrow$ implies $\mathfrak{C}\left[\llbracket \mathrm{t}_{2} \rrbracket\right] \Downarrow$. In a sufficiently-powerful source language, we can construct a backtranslation $\langle\langle\mathfrak{C}\rangle\rangle$ for any target-level context $\mathfrak{C}$. Using the same logical relation as above, it then suffices to prove that $\langle\langle\mathfrak{C}\rangle\rangle$ is a valid source-level context and that $\langle\langle\mathfrak{C}\rangle\rangle \approx \mathfrak{C}$ for any valid context $\mathfrak{C}$. Together with $t_{1} \approx \llbracket t_{1} \rrbracket$, and similarly for $t_{2}$, compositionality

\footnotetext{
${ }^{1}$ Actually, both Figs. 2 and 3 are simplifications. Perceptive readers may notice that the proof depicted here would falsely imply equivalence preservation for any correct compiler. We correct the simplifications in Section 5.6.
}

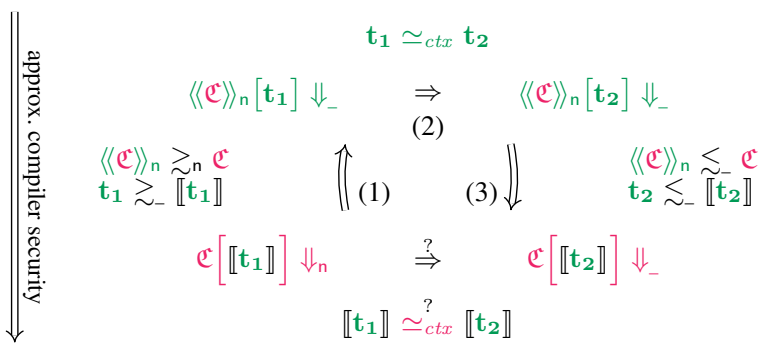

Figure 3. Proving equivalence preservation using an $n$ approximate back-translation. An _ subscript indicates any number of steps.

and adequacy of the logical relation then yield implications (1) and (3) in the figure. The remaining implication (2) follows from the assumed (contextual) equivalence of $t_{1}$ and $t_{2}$.

Constructing a back-translation of contexts is not easy, but it can be done if the source language is sufficiently expressive. Consider, for example, a compiler that translates terms from a simply-typed $\lambda$-calculus with recursive types $\left(\lambda^{\tau ; \mu}\right)$ to an untyped $\lambda$-calculus $\left(\lambda^{\mathrm{u}}\right)$. Constructing a back-translation of target-level contexts can be done based on a $\lambda^{\tau ; \mu}$ type that can represent arbitrary $\lambda^{\mathrm{u}}$ values. Particularly, we can encode the unitype of $\lambda^{u}$ values in a type UVal as follows:

$$
\mathrm{UVal} \stackrel{\text { def }}{=} \mu \alpha \cdot \mathcal{B} \uplus(\alpha \times \alpha) \uplus(\alpha \uplus \alpha) \uplus(\alpha \rightarrow \alpha)
$$

given that $\lambda^{u}$ has base values of type $\mathcal{B}$, pairs, coproducts and lambdas. In other words, all $\lambda^{\mathrm{u}}$ values can be represented as $\lambda^{\tau ; \mu}$ values of type UVal. We can then construct a back-translation of $\lambda^{u}$ contexts to $\lambda^{\tau ; \mu}$ contexts such that the latter work with values in UVal wherever the original $\lambda^{\mathrm{u}}$ context worked with arbitrary $\lambda^{\mathrm{u}}$ values.

Contributions of this paper If the types of the source language are not powerful enough to embed an encoding of target terms, is it possible to have a fully-abstract compiler between those languages? In this paper we answer positively to this question and develop a general technique for proving this. We instantiate this proof technique and develop a fully-abstract compiler from a simply-typed $\lambda$-calculus without recursive types $\left(\lambda^{\tau}\right)$ to an untyped $\lambda$-calculus $\left(\lambda^{\mathrm{u}}\right)$. With such a source language, we cannot construct a type like UVal to represent the values that a $\lambda^{u}$ context works with. Fortunately, we can solve this problem by observing that a fully accurate emulation is sufficient for the proof but in fact not necessary. An approximate back-translation is enough for the full-abstraction proof to work, without sacrificing the overall simplicity and elegance of the proof technique. The basic idea is depicted in Fig. 3. The differences from Fig. 2 are the use of asymmetric logical relations $\lesssim$ and $\gtrsim$ (also known as logical approximations) to express (roughly) that a term (or context) $t$ terminates whenever $t$ does $(t \gtrsim t)$ and vice versa ( $\mathrm{t} \lesssim \mathrm{t}$ ) and the addition of subscripts $n$ where logical approximations hold only up to a limited number of steps $n$. Note that $n$ in the Figure is defined as the number of steps in the evaluation $\mathfrak{C}\left[\llbracket \mathrm{t}_{1} \rrbracket\right] \Downarrow_{n}$ and that we write for an unknown number of steps.

The proof starts, again, from an arbitrary target-level context $\mathfrak{C}$ and the knowledge that $\mathfrak{C}\left[\llbracket \mathrm{t}_{1} \rrbracket\right] \Downarrow_{n}$ and we call $n$ the number of reduction steps in this execution. We then construct a $\lambda^{\tau}$ context $\left\langle\langle\mathfrak{C}\rangle_{\mathrm{n}}\right.$ that satisfies two conditions. First, it approximates $\mathfrak{C}$ up to $n$ steps: $\langle\langle\mathfrak{C}\rangle\rangle_{\mathrm{n}} \gtrsim_{\mathrm{n}} \mathfrak{C}$. This means that if $\mathfrak{C}[\mathrm{t}]$ terminates in less than $n$ steps then $\langle\langle\mathfrak{C}\rangle\rangle_{\mathrm{n}}[\mathrm{t}]$ will also terminate for a term $\mathrm{t}$ related to $t$. This, together with the knowledge that $t \gtrsim \llbracket t \rrbracket$, allows us to deduce implication (1) in the figure. As before, implication (2) follows directly from the (contextual) equivalence of $t_{1}$ and $t_{2}$. 
Then we use a second condition on the $n$-approximation $\langle\langle\mathfrak{C}\rangle\rangle_{n}$, namely that it has to be conservative, to deduce implication (3). Intuitively, the source-level context produced by the $n$-approximation may diverge in situations where the original did not, but not vice versa. Intuitively, the divergence will occur when the precision $n$ of approximate back-translation $\langle\langle\mathfrak{C}\rangle\rangle_{\mathrm{n}}$ is not sufficient for the context to accurately simulate the behavior of $\mathfrak{C}$. This is expressed by the logical approximation $\langle\langle\mathfrak{C}\rangle\rangle_{\mathrm{n}} \lesssim \mathfrak{C}$ which implies that if $\langle\langle\mathfrak{C}\rangle\rangle_{\mathrm{n}}[\mathrm{t}]$ terminates (in any number of steps), then so must $\mathfrak{C}[t]$. This allows us to deduce implication (3).

The advantage of this approximate back-translation approach is that it can be easier to construct a conservative approximate backtranslation than a full one. For example, considering $\lambda^{\tau}$ without recursive types, we can construct a family of $\lambda^{\tau}$ types $\mathrm{UVal}_{n}$, indexed by non-negative numbers $n$ :

$$
\begin{aligned}
& \mathrm{UVal}_{0} \stackrel{\text { def }}{=} \text { Unit } \\
& \mathrm{UVal}_{n+1} \stackrel{\text { def }}{=} \operatorname{Unit} \uplus \mathcal{B} \uplus\left(\mathrm{UVal}_{n} \times \mathrm{UVal}_{n}\right) \uplus\left(\mathrm{UVal}_{n}\right. \\
&\left.\uplus \mathrm{UVal}_{n}\right) \uplus\left(\mathrm{UVal}_{n} \rightarrow \mathrm{UVal}_{n}\right) .
\end{aligned}
$$

Without giving full details here, $\mathrm{UVal}_{\mathrm{n}}$ is an $n$-level unfolding of UVal with additional unit values at every level to represent failed approximations. This approximate version of UVal is enough to construct a conservative $n$-approximate back-translation of an untyped program context, and as such, it allows us to circumvent the lack of expressiveness of $\lambda^{\tau}$ without recursive types.

In order to make this approximate back-translation approach work, we need a way to formalise the relation between an untyped context and its approximate back-translation. However, it turns out that existing well-known techniques from the field of logical relations are almost directly applicable. Asymmetric logical relations (like $\langle\langle\mathfrak{C}\rangle\rangle_{\mathrm{n}} \lesssim \mathfrak{C}$ above) are a well-established technique. More interestingly, the approximateness of the relation can very naturally be expressed using step-indexed logical relations. Despite this naturality, it appears that this use of step-indexing is novel. The technique is normally used as a way to construct well-founded logical relations and one is not actually interested in terms being related only up to a limited number of steps.

To summarise, the contributions of this work are:

- a new and general proof technique for proving compiler fullabstraction using asymmetric, cross-language logical relations and targeting untyped languages;

- an instantiation of that proof technique to fully-abstractly compile a simply-typed $\lambda$-calculus without recursive types to the untyped $\lambda$-calculus;

- a novel application of step-indexed logical relations for expressing approximateness of a back-translation.

This paper is structured as follows. Firstly it formalises the source and target languages $\lambda^{\tau}$ and $\lambda^{\mathrm{u}}$ (Section 2). Secondly it presents the cross-language logical relations that are used to express the relation between $\lambda^{\tau}$ terms and their compilations as well as between $\lambda^{\mathrm{u}}$ contexts and their back-translation (Section 3 ). We define the compiler in Section 4. It applies type erasure and dynamic type wrappers that enforce the requirements and guarantees of $\lambda^{\tau}$ types during execution. The paper then presents the approximate back-translation (Section 5) which is used when proving compiler full-abstraction (Section 6). Finally, we discuss the presented results (Section 7), related work (Section 8) and we conclude in Section 9.

\section{Source and Target Languages}

The source language $\lambda^{\tau}$ is presented in Fig. 4. It is a standard, strict, simply-typed $\lambda$-calculus with Unit, Bool, lambdas, product and sum types and a fix operator providing general recursion. The figure presents the syntax of terms $\mathbf{t}$, values $\mathbf{v}$, types $\tau$, typing contexts $\Gamma$ and evaluation contexts $\mathbb{C}$. Apart from the type and evaluation rules for fix $_{\tau_{1} \rightarrow \tau_{2}}$, the typing rules and evaluation rules are standard. The evaluation rules use evaluation contexts to impose a strict evaluation order. The type and evaluation rule for fix $x_{\tau_{1} \rightarrow \tau_{2}}$ are somewhat special compared to a more standard definition (see e.g. [Pierce, 2002]): the operator is restricted to function types and an additional $\eta$-expansion occurs during evaluation. This is because we have chosen to make fix model the $\mathrm{Z}$ fixed-point combinator (also known as the call-by-value Y combinator) [Pierce, 2002, §5] rather than the Y combinator. The reason revolves around the compiler devised in this paper. The target language of that compiler is a strict untyped lambda calculus, where Y does not work but Z does and using $\mathrm{Z}$ in $\lambda^{\tau}$ as well keeps the compiler simpler. Working with the more standard Y fixpoint combinator in $\lambda^{\tau}$ is probably possible but would require the compiler to use an encoding that would be pervasive but irrelevant to the subject of this paper.

$\lambda^{\tau}$ program contexts $\mathfrak{C}$ are $\lambda^{\tau}$ terms that contain exactly one hole [.] in place of a subterm (we omit the formal definition). We also omit the typing judgement for program contexts $\vdash \mathfrak{C}$ : $\Gamma^{\prime}, \tau^{\prime} \rightarrow \Gamma, \tau$, defined by inductive rules close to those for terms in Fig. 4. The judgement guarantees that substituting a well-typed term $\Gamma^{\prime} \vdash \mathrm{t}: \tau^{\prime}$ in a well-typed context $\vdash \mathfrak{C}: \Gamma^{\prime}, \tau^{\prime} \rightarrow \Gamma, \tau$ produces a well-typed term $\Gamma \vdash \mathfrak{C}[\mathrm{t}]: \tau$.

Figure 5 presents the syntax, well-scopedness and evaluation rules for the target language $\lambda^{u}$ : a standard untyped $\lambda$-calculus. The calculus has unit, booleans, lambdas, product and sum values, and produces a kind of unrecoverable exception in case of type errors (e.g. projecting from a non-pair value, case splitting on a non-sum value etc.). Such an unrecoverable exception is represented in a standard way (see, e.g., [Pierce, 2002, §14.1]) as a non-value term wrong with a special reduction rule. The well-scopedness rules are unsurprising and the evaluation rules again use evaluation contexts to impose a strict evaluation order. Note that the termination judgement $t \Downarrow$ requires termination with a value, i.e. not wrong. Again, we omit the definition of program contexts $\mathfrak{C}$ (expressions with a single hole in place of a subterm) and their well-scopedness judgement $\vdash \mathfrak{C}: \Gamma^{\prime} \rightarrow \Gamma$, whose inductive definition guarantees that substituting a well-scoped term $\Gamma^{\prime} \vdash \mathrm{t}$ for the hole produces a wellscoped result term $\Gamma \vdash \mathfrak{C}[\mathrm{t}]$.

The interested reader can find full formalisation and proofs in a separately-published technical appendix [Devriese et al., 2016].

\section{Logical Relations}

This section presents the Kripke, step-indexed logical relations that are used to prove compiler full-abstraction. Firstly, this section describes the specifications of the world used by the logical relation (Fig. 6). Then, it defines the logical relations (Fig. 7) and finally it proves standard properties that the relations enjoy. Note that part of these logical relations, namely the novel insights needed to provide compiler full-abstraction, are postponed until Section 5.2. The goal of this section is to provide an understanding of what it means for two terms to be related; this will be needed for understanding properties of the compiler in the following sections.

The cross-language logical relations used in this paper are roughly based on one by Hur and Dreyer [2011]. Essentially, we instantiate their language-generic logical relations to $\lambda^{\tau}$ and $\lambda^{\mathrm{u}}$ and simplify them by removing complexities deriving from the System F type system, public/private transitions, references and garbage collection.

Since we do not deal with mutable references, we use a very simple notion of worlds, consisting just of a step-index $k$ that can be accessed with the lev $(\cdot)$ function (Fig. 6). We define a $\triangleright$ modal- 


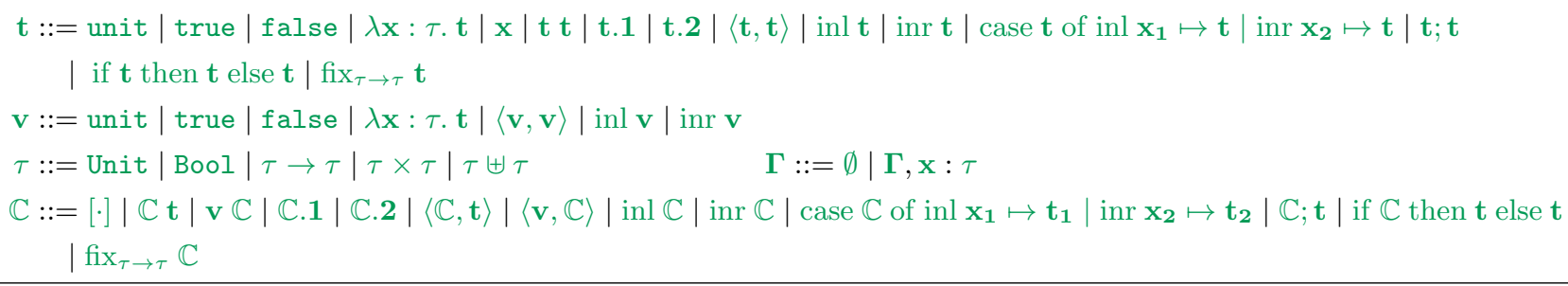

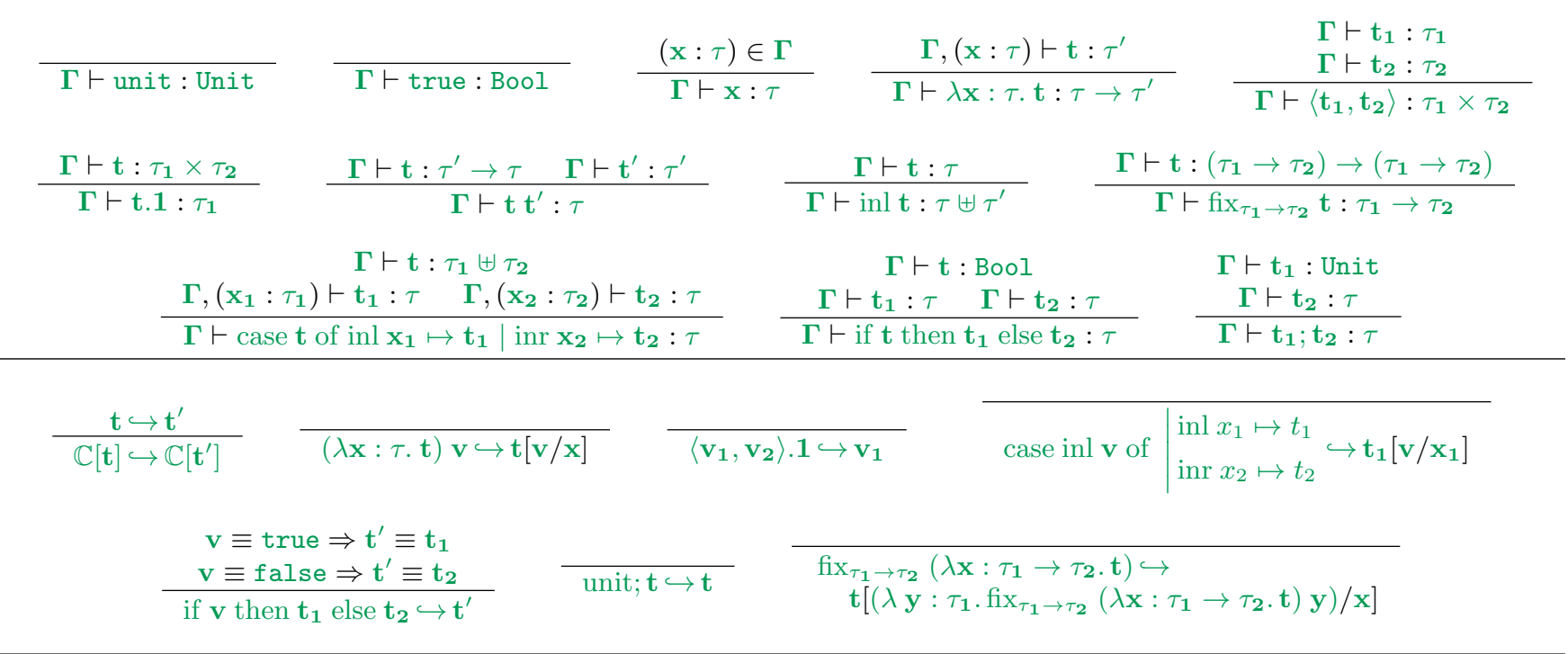

Figure 4. Syntax, static and dynamic semantics of the source language $\lambda^{\tau}$ (selection of).

$\mathrm{t}::=$ unit $\mid$ true $\mid$ false $|\lambda x . t| x|t \mathrm{t}| \mathrm{t} .1|\mathrm{t} .2|\langle\mathrm{t}, \mathrm{t}\rangle \mid$ inl $\mathrm{t} \mid$ inr $\mathrm{t} \mid$ case $\mathrm{t}$ of inl $\mathrm{x} \mapsto \mathrm{t} \mid$ inr $\mathrm{x} \mapsto \mathrm{t} \mid \mathrm{t}$; $\mathrm{t} \mid$ if $\mathrm{t}$ then $\mathrm{t}$ else $\mathrm{t} \mid$ wrong $v::=$ unit $\mid$ true $\mid$ false $|\lambda x . t|\langle v, v\rangle|\operatorname{inl} v| \operatorname{inr} v \quad \Gamma::=\emptyset \mid \Gamma, x$ $\mathbb{C}::=[\cdot]|\mathbb{C} \mathrm{t}| \vee \mathbb{C}|\mathbb{C} .1| \mathbb{C} .2|\langle\mathbb{C}, \mathrm{t}\rangle|\langle\mathrm{v}, \mathbb{C}\rangle|\operatorname{inl} \mathbb{C}| \operatorname{inr} \mathbb{C} \mid$ case $\mathbb{C}$ of inl $\mathrm{x}_{1} \mapsto \mathrm{t}_{1} \mid$ inr $\mathrm{x}_{2} \mapsto \mathrm{t}_{2} \mid \mathbb{C}$; $\mathrm{t} \mid$ if $\mathbb{C}$ then $\mathrm{t}$ else $\mathrm{t}$

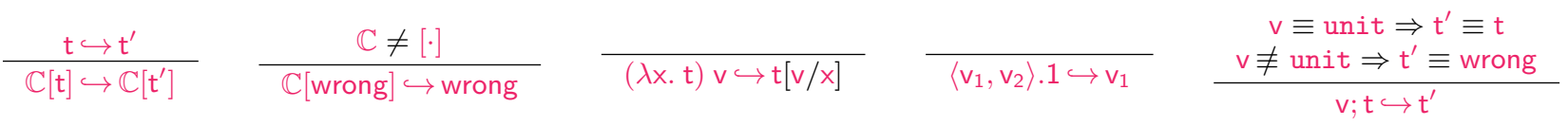

$$
\begin{aligned}
& \text { case inl v of } \mid \begin{array}{l}
\operatorname{inl} x_{1} \mapsto t_{1} \\
\operatorname{inr} x_{2} \mapsto t_{2}
\end{array} \hookrightarrow \mathrm{t}_{1}\left[\mathrm{v} / \mathrm{x}_{1}\right]
\end{aligned}
$$

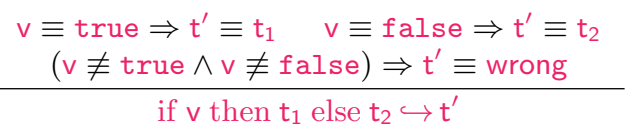

Figure 5. Syntax and dynamic semantics of the target language $\lambda^{\mathrm{u}}$ (selection of).

$$
\begin{array}{cc}
\underline{\mathrm{W}}::=(k) \text { with } k \in \mathbb{N} & \triangleright(k+1) \stackrel{\text { def }}{=}(k) \\
\operatorname{lev}(\underline{\mathrm{W}}) \stackrel{\text { def }}{=} \underline{\mathrm{W}} \cdot k & (k) \sqsupseteq\left(k^{\prime}\right) \stackrel{\text { def }}{=} k \leq k^{\prime} \\
\triangleright(0) \stackrel{\text { def }}{=}(0) & (k) \sqsupset_{\triangleright}\left(k^{\prime}\right) \stackrel{\text { def }}{=} k<k^{\prime} \\
\left.\mathrm{O}(\underline{\mathrm{W}})_{\lesssim} \stackrel{\text { def }}{=}\left\{(\mathrm{t}, \mathrm{t}) \mid \exists k \leq \operatorname{lev}(\underline{\mathrm{W}}), \mathrm{v} \cdot \mathrm{t} \hookrightarrow^{\mathrm{k}} \mathrm{v} \Rightarrow \exists k^{\prime}, \mathrm{v} \cdot \mathrm{t} \hookrightarrow^{\mathrm{k}^{\prime}} \mathrm{v}\right)\right\} \\
\left.\mathrm{O}(\underline{\mathrm{W}})_{\gtrsim} \stackrel{\text { def }}{=}\left\{(\mathrm{t}, \mathrm{t}) \mid \exists k \leq \operatorname{lev}(\underline{\mathrm{W}}), \mathrm{v} \cdot \mathrm{t} \hookrightarrow^{\mathrm{k}} \mathrm{v} \Rightarrow \exists k^{\prime}, \mathrm{v} \cdot \mathrm{t} \hookrightarrow^{\mathrm{k}^{\prime}} \mathrm{v}\right)\right\}
\end{array}
$$

Figure 6. Logical relations: Worlds. ity and a future world relation $\sqsupseteq$, expressing that future worlds allow less reduction steps to be taken. We define two different observation relations $\mathrm{O}(\underline{\mathrm{W}})_{\lesssim}$ and $\mathrm{O}(\underline{\mathrm{W}})_{\gtrsim}$. The former defines that a $\lambda^{\tau}$ term $\mathrm{t}$ approximates a $\lambda^{\mathrm{u}}$ term $\mathrm{t}$ if termination of the first in less than $\operatorname{lev}(\underline{W})$ steps implies termination of the second (in an unknown number of steps). The latter requires the reverse. All of our logical relations will be defined in terms of either $\mathrm{O}(\underline{\mathrm{W}}) \lesssim$ or $\mathrm{O}(\underline{\mathrm{W}})_{\gtrsim}$. For definitions and lemmas or theorems that apply for both instantiations, we use the symbol $\square$ as a metavariable that can be instantiated to either $\lesssim$ and $\gtrsim$.

Figure 7 contains the definition of the logical relations. The first thing to note is that our logical relations are not indexed by $\lambda^{\tau}$ types $\tau$, but by pseudo-types $\hat{\tau}$. The syntax for these pseudotypes contains all the constructs of $\lambda^{\tau}$ types, plus an additional 
Pseudo-types $\hat{\tau}$, pseudo-contexts $\hat{\Gamma}$, oftype $(\cdot)$ and repEmul $(\cdot)$.

$$
\begin{aligned}
& \hat{\tau}::=\text { Bool } \mid \text { Unit }|\hat{\tau} \times \hat{\tau}| \hat{\tau} \uplus \hat{\tau}|\hat{\tau} \rightarrow \hat{\tau}| \text { EmulDV }_{\mathrm{n} ; \mathrm{p}} \quad \hat{\Gamma}::=\emptyset \mid \hat{\Gamma}, \mathbf{x}: \hat{\tau} \\
& \operatorname{repEmul}(\hat{\tau}) \stackrel{\text { def }}{=} \ldots \text { (to be defined later, in Fig. 12) } \\
& \operatorname{oftype}(\hat{\tau}) \stackrel{\text { def }}{=}\{\mathrm{v} \mid \emptyset \vdash \mathrm{v}: \operatorname{repEmul}(\hat{\tau})\}
\end{aligned}
$$

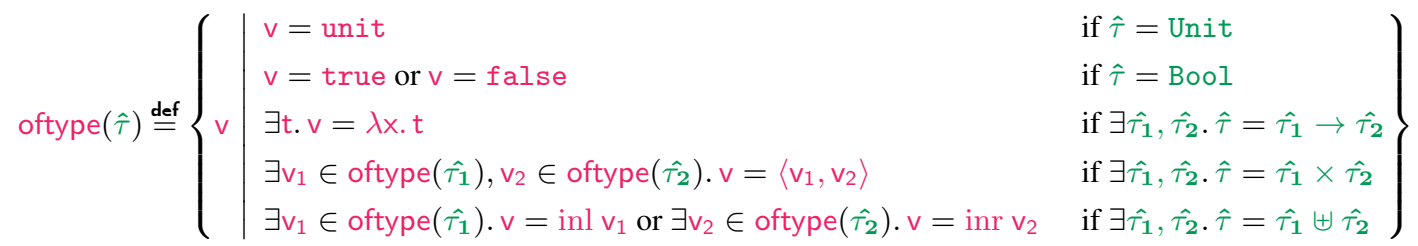

$$
\begin{aligned}
& \operatorname{oftype}(\hat{\tau}) \stackrel{\text { def }}{=}\{(\mathrm{v}, \mathrm{v}) \mid \mathrm{v} \in \operatorname{oftype}(\hat{\tau}) \wedge \mathrm{v} \in \operatorname{oftype}(\hat{\tau})\}
\end{aligned}
$$

Logical relations for values $(\mathcal{V} \llbracket \rrbracket)$, contexts $(\mathcal{K} \llbracket \cdot \rrbracket)$, terms $(\mathcal{E} \llbracket \cdot \rrbracket)$ and environments $(\mathcal{G} \llbracket \rrbracket)$.

$$
\begin{aligned}
& \triangleright R \stackrel{\text { def }}{=}\{(\underline{\mathrm{W}}, \mathrm{v}, \mathrm{v}) \mid \operatorname{lev}(\underline{\mathrm{W}})>0 \Rightarrow(\triangleright \underline{\mathrm{W}}, \mathrm{v}, \mathrm{v}) \in R\} \\
& \mathcal{V} \llbracket \text { Unit } \rrbracket \stackrel{\text { def }}{=}\{(\underline{\mathrm{W}}, \mathrm{v}, \mathrm{v}) \mid \mathrm{v}=\text { unit and } \mathrm{v}=\text { unit }\} \\
& \mathcal{V} \llbracket \text { Bool } \rrbracket_{\square} \stackrel{\text { def }}{=}\{(\underline{\mathrm{W}}, \mathrm{v}, \mathrm{v}) \mid \exists v \in\{\text { true, false }\} . \mathrm{v}=v \text { and } \mathrm{v}=v\} \\
& \mathcal{V} \llbracket \hat{\tau}^{\prime} \rightarrow \hat{\tau} \rrbracket_{\square} \stackrel{\text { def }}{=}\left\{(\underline{\mathbf{W}}, \mathrm{v}, \mathrm{v}) \mid \begin{array}{r}
(\mathrm{v}, \mathrm{v}) \in \operatorname{oftype}\left(\hat{\tau^{\prime}} \rightarrow \hat{\tau}\right) \text { and } \exists \mathrm{t}, \mathrm{t} . \mathrm{v}=\lambda \mathrm{x}: \operatorname{repEmul}\left(\hat{\tau^{\prime}}\right) . \mathrm{t} \text { and } \mathrm{v}=\lambda \mathrm{x} . \mathrm{t} \text { and } \\
\forall \underline{\mathbf{W}^{\prime}} \sqsupset_{\triangleright} \underline{\mathrm{W}},\left(\underline{\mathbf{W}^{\prime}}, \mathrm{v}^{\prime}, \mathrm{v}^{\prime}\right) \in \mathcal{V} \llbracket \hat{\tau}^{\prime} \rrbracket \square \cdot\left(\underline{\mathbf{W}^{\prime}}, \mathrm{t}\left[\mathrm{v}^{\prime} / \mathrm{x}\right], \mathrm{t}\left[\mathrm{v}^{\prime} / \mathrm{x}\right]\right) \in \mathcal{E} \llbracket \hat{\tau} \rrbracket \square
\end{array}\right\} \\
& \mathcal{V} \llbracket \hat{\tau_{1}} \times \hat{\tau_{2}} \rrbracket_{\square} \stackrel{\text { def }}{=}\left\{(\underline{\mathrm{W}}, \mathrm{v}, \mathrm{v}) \mid \begin{array}{r}
(\mathrm{v}, \mathrm{v}) \in \operatorname{oftype}\left(\hat{\tau_{1}} \times \hat{\tau_{2}}\right) \text { and } \exists \mathrm{v}_{1}, \mathrm{v}_{2}, \mathrm{v}_{1}, \mathrm{v}_{2} \cdot \mathrm{v}=\left\langle\mathrm{v}_{1}, \mathrm{v}_{2}\right\rangle \text { and } \mathrm{v}=\left\langle\mathrm{v}_{1}, \mathrm{v}_{2}\right\rangle \text { and } \\
\left(\underline{\mathrm{W}}, \mathrm{v}_{1}, \mathrm{v}_{1}\right) \in \triangleright \mathcal{V} \llbracket \hat{\tau_{1}} \rrbracket \square \text { and }\left(\underline{\mathrm{W}}, \mathrm{v}_{2}, \mathrm{v}_{2}\right) \in \triangleright \mathcal{V} \llbracket \hat{\tau_{2}} \rrbracket \square
\end{array}\right\}
\end{aligned}
$$

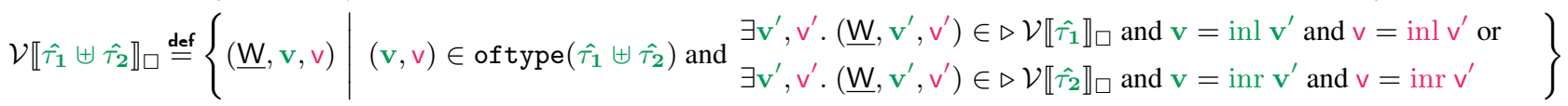

$\mathcal{V} \llbracket E m u l D V_{\mathrm{n} ; \mathrm{p}} \rrbracket \square \stackrel{\text { def }}{=} \ldots$ (to be defined later, in Fig. 11)

$$
\begin{aligned}
& \mathcal{K} \llbracket \hat{\tau} \rrbracket_{\square} \stackrel{\text { def }}{=}\left\{(\underline{\mathrm{W}}, \mathbb{C}, \mathbb{C}) \mid \forall \underline{\mathrm{W}}^{\prime} \sqsupseteq \underline{\mathrm{W}},\left(\underline{\mathrm{W}}^{\prime}, \mathrm{v}, \mathrm{v}\right) \in \mathcal{V} \llbracket \hat{\tau} \rrbracket_{\square} \cdot(\mathbb{C}[\mathrm{v}], \mathbb{C}[\mathrm{v}]) \in \mathrm{O}\left(\underline{\mathrm{W}}^{\prime}\right)_{\square}\right\} \\
& \mathcal{E} \llbracket \hat{\tau} \rrbracket_{\square} \stackrel{\text { def }}{=}\left\{(\underline{\mathrm{W}}, \mathrm{t}, \mathrm{t}) \mid \forall(\underline{\mathrm{W}}, \mathbb{C}, \mathbb{C}) \in \mathcal{K} \llbracket \hat{\tau} \rrbracket_{\square} \cdot(\mathbb{C}[\mathrm{t}], \mathbb{C}[\mathrm{t}]) \in \mathrm{O}(\underline{\mathrm{W}})_{\square}\right\}
\end{aligned}
$$

Logical relations for substitutions, open terms and program contexts.

$$
\begin{array}{r}
\mathcal{G} \llbracket \emptyset \rrbracket_{\square} \stackrel{\text { def }}{=}\{(\underline{\mathrm{W}}, \emptyset, \emptyset)\} \\
\mathcal{G} \llbracket \hat{\Gamma},(\mathrm{x}: \hat{\tau}) \rrbracket_{\square} \stackrel{\text { def }}{=}\left\{(\underline{\mathrm{W}}, \gamma[\mathrm{x} \mapsto \mathrm{v}], \gamma[\mathrm{x} \mapsto \mathrm{v}]) \mid(\underline{\mathrm{W}}, \gamma, \gamma) \in \mathcal{G} \llbracket \hat{\Gamma} \rrbracket_{\square} \text { and }(\underline{\mathrm{W}}, \mathrm{v}, \mathrm{v}) \in \mathcal{V} \llbracket \hat{\tau} \rrbracket_{\square}\right\} \\
\hat{\Gamma} \vdash \mathrm{t} \square_{\mathrm{n}} \mathrm{t}: \hat{\tau} \stackrel{\text { def }}{=} \operatorname{repEmul}(\hat{\Gamma}) \vdash \mathrm{t}: \operatorname{repEmul}(\hat{\tau}) \text { and } \underline{\underline{\mathrm{W}}} \cdot \operatorname{lev}(\underline{\mathrm{W}}) \leq n \Rightarrow \forall(\underline{\mathrm{W}}, \gamma, \gamma) \in \mathcal{G} \llbracket \hat{\Gamma} \rrbracket_{\square} \cdot(\underline{\mathrm{W}}, \mathrm{t} \gamma, \mathrm{t} \gamma) \in \mathcal{E} \llbracket \hat{\tau} \rrbracket_{\square} \\
\hat{\Gamma} \vdash \mathrm{t} \square \mathrm{t}: \hat{\tau} \stackrel{\text { def }}{=} \hat{\Gamma} \vdash \mathrm{t} \square_{\mathrm{n}} \mathrm{t}: \hat{\tau} \text { for all } n \\
\vdash \mathfrak{C} \square_{\mathrm{n}} \mathfrak{C}: \hat{\Gamma}^{\prime}, \hat{\tau}^{\prime} \rightarrow \hat{\Gamma}, \hat{\tau} \stackrel{\text { def }}{=} \vdash \mathfrak{C}: \operatorname{repEmul}\left(\hat{\Gamma}^{\prime}\right), \operatorname{repEmul}\left(\hat{\tau}^{\prime}\right) \rightarrow \operatorname{repEmul}(\hat{\Gamma}), \operatorname{repEmul}(\hat{\tau}) \text { and } \\
\quad \text { for all } \mathrm{t}, \mathrm{t} . \text { if } \hat{\Gamma}^{\prime} \vdash \mathrm{t} \square_{\mathrm{n}} \mathrm{t}: \hat{\tau}^{\prime}, \text { then } \hat{\Gamma} \vdash \mathfrak{C}[\mathrm{t}] \square_{\mathrm{n}} \mathfrak{C}[\mathrm{t}]: \hat{\tau}
\end{array}
$$

Figure 7. Logical relations (partial, the missing definition can be found in Figs. 11 and 12).

kind of token type EmulDV $V_{n ; p}$, indexed by a non-negative number $n$ and a value $p::=$ precise | imprecise. This token type is not a $\lambda^{\tau}$ type; it is needed because of the approximate backtranslation. When necessary, we use a function repEmul() for converting a pseudo-type to a $\lambda^{\tau}$ type. The function replaces all occurrences of EmulDV $V_{n ; p}$ with a concrete $\lambda^{\tau}$ type. We postpone the definitions and explanations of EmulDV $V_{n ; p}$ and of $\mathcal{V} \llbracket E m u l D V_{n ; p} \rrbracket_{\square}$ to Section 5.2, after we have given some more information about the back-translation. We will sometimes silently use a normal type where a pseudo-type is expected, which makes sense since the syntax for the latter is a superset of the former.

The value relation $\mathcal{V} \llbracket \hat{\tau} \rrbracket_{\square}$ is defined by induction on the pseudotype. Most definitions are quite standard. All cases require related terms to be in the of type relation, which requires well-typedness of the $\lambda^{\tau}$ term and an appropriate shape for the $\lambda^{u}$ value. Unit and Bool values are related in any world iff they are the same base value. Pair values are related if both are pairs and the corresponding components are related in strictly future worlds at the appropriate pseudo-type. Similarly, sum values are related if they are both of either the form inl $\cdots$ or inr $\cdots$ and if the contained values are related in strictly future worlds at the appropriate pseudo-type. Finally, function values are related if they have the right type, if both are lambdas and if substituting related values in the body yields related terms in any strictly future world.

The relation on values, evaluation contexts and terms are defined mutually recursively, using a technique known as biorthogonality (see, e.g., Benton and Hur [2009]). So, evaluation contexts are related in a world if plugging in related values in any future 


$$
\begin{aligned}
& f i x \stackrel{\text { def }}{=} \lambda f .(\lambda x \cdot f(\lambda y \cdot x x y))(\lambda x \cdot f(\lambda y \cdot x \times y)) \\
& \operatorname{erase}\left(u_{n i t}\right) \stackrel{\text { def }}{=} \text { unit } \operatorname{erase}\left(\left\langle\mathrm{t}_{1}, \mathrm{t}_{2}\right\rangle\right) \stackrel{\text { def }}{=}\left\langle\operatorname{erase}\left(\mathrm{t}_{1}\right), \operatorname{erase}\left(\mathrm{t}_{2}\right)\right\rangle \\
& \operatorname{erase}(f a l s e) \stackrel{\text { def }}{=} \text { false } \operatorname{erase}\left(\mathrm{t}_{1} ; \mathrm{t}_{2}\right) \stackrel{\text { def }}{=} \operatorname{erase}\left(\mathrm{t}_{1}\right) ; \operatorname{erase}\left(\mathrm{t}_{2}\right) \\
& \operatorname{erase}(\mathrm{x}) \stackrel{\text { def }}{=} \mathrm{x} \quad \operatorname{erase}\left(\mathrm{t}_{1} \mathrm{t}_{2}\right) \stackrel{\text { def }}{=} \operatorname{erase}\left(\mathrm{t}_{1}\right) \operatorname{erase}\left(\mathrm{t}_{2}\right) \\
& \operatorname{erase}(\mathrm{t} .1) \stackrel{\text { def }}{=} \operatorname{erase}(\mathrm{t}) .1 \\
& \operatorname{erase}(\mathrm{t} .2) \stackrel{\text { def }}{=} \operatorname{erase}(\mathrm{t}) .2 \\
& \operatorname{erase}(\mathrm{inl} \mathrm{t}) \stackrel{\text { def }}{=} \mathrm{inl} \text { erase }(\mathrm{t}) \\
& \text { erase (inr } \mathrm{t}) \stackrel{\text { def }}{=} \text { inr erase }(\mathrm{t}) \\
& \text { erase (if } \left.t \text { then } t_{1} \text { else } t_{2}\right) \stackrel{\text { def }}{=} \text { if erase }(t) \text { then erase }\left(t_{1}\right) \\
& \text { erase (case } \left.\mathrm{t} \text { of inl } \mathrm{x}_{1} \mapsto \mathrm{t}_{1} \mid \text { inr } \mathrm{x}_{2} \mapsto \mathrm{t}_{2}\right) \stackrel{\text { def }}{=} \\
& \text { erase(true) } \stackrel{\text { def }}{=} \text { true } \\
& \operatorname{erase}(\lambda \mathbf{x}: \tau . \mathbf{t}) \stackrel{\text { def }}{=} \lambda \mathrm{x} \cdot \operatorname{erase}(\mathrm{t}) \\
& \operatorname{erase}\left(\text { fix }_{\tau_{1} \rightarrow \tau_{2}} \mathrm{t}\right) \stackrel{\text { def }}{=} f i x \operatorname{erase}(\mathrm{t}) \\
& \text { else erase }\left(t_{2}\right) \\
& \operatorname{case} \operatorname{erase}(t) \text { of } \mid \begin{array}{l}
\operatorname{inl} x_{1} \mapsto \operatorname{erase}\left(t_{1}\right) \\
\operatorname{inr} x_{2} \mapsto \operatorname{erase}\left(t_{2}\right)
\end{array}
\end{aligned}
$$

Figure 8. Type erasure: the first pass of the compiler.

world yields related observations. Similarly, terms are related if plugging the terms in related evaluation contexts yields related observations. Relation $\mathcal{G} \llbracket \Gamma \rrbracket_{\square}$ relates substitutions instantiating a context $\Gamma$, which simply requires that substitutions for all variables in the context are related at their types. For open terms, we define a logical relation $\hat{\Gamma} \vdash \mathrm{t} \square_{\mathrm{n}} \mathrm{t}: \hat{\tau}$. This relation expresses that an open $\lambda^{\tau}$ term $\mathrm{t}$ is related up to $n$ steps to an open $\lambda^{\mathrm{u}}$ term $\mathrm{t}$ at pseudo-type $\hat{\tau}$ in pseudo-context $\hat{\Gamma}$ if the first is well-typed and if closing $t$ and $t$ with substitutions related at pseudo-context $\hat{\Gamma}$ produces terms related at pseudo-type $\hat{\tau}$, in any world $\underline{\mathrm{W}}$ such that $\operatorname{lev}(\underline{\mathrm{W}}) \leq n$. If $\hat{\Gamma} \vdash \mathrm{t} \square_{\mathrm{n}} \mathrm{t}: \hat{\tau}$ for any $n$, then we write $\hat{\Gamma} \vdash \mathrm{t} \square \mathrm{t}: \hat{\tau}$. Finally, we define a logical relation for program contexts $\vdash \mathfrak{C} \square \mathfrak{C}: \hat{\Gamma}^{\prime}, \hat{\tau}^{\prime} \rightarrow \hat{\Gamma}, \hat{\tau}$ which requires that substituting terms related at the appropriate pseudo-type type produces terms related at the appropriate pseudo-type.

It is interesting to note that the simple type system of our source calculus does not actually present a technical need for the use of step-indexing. Because there are no recursive types or general references, it is a simple enough system that we can give wellfounded logical relations without any step-indexing. However, as mentioned before, we use step-indexing for a different reason than other work: not for constructing a well-founded logical relation, but for stating that two terms are related only up to a certain number of steps. More details follow in Section 5.

These logical relations are constructed so that termination of one implies termination of the other, according to the direction of the approximation $(\lesssim$ or $\gtrsim$, Lemma 1$)$.

Lemma 1 (Adequacy for $\lesssim$ and $\gtrsim$ ). If $\emptyset \vdash \mathrm{t} \lesssim \mathrm{n} t: \tau$, and if $\mathrm{t} \hookrightarrow^{\mathrm{m}} \mathrm{v}$ with $n \geq m$, then also $\mathrm{t} \Downarrow$. If $\emptyset \vdash \mathrm{t} \gtrsim_{\mathrm{n}} \mathrm{t}: \tau$ and if $\mathrm{t} \hookrightarrow^{\mathrm{m}} \vee$ with $n \geq \bar{m}$, then also $\mathrm{t} \Downarrow$.

\section{The Compiler}

This section presents our compiler from $\lambda^{\tau}$ to $\lambda^{\mathrm{u}}$. The compiler proceeds in two passes: type erasure (Fig. 8) and dynamic typechecking wrappers (Fig. 9).

The erasure function is called erase; it converts all $\lambda^{\tau}$ constructs to the corresponding $\lambda^{\mathrm{u}}$ constructs. fix $x_{\tau_{1} \rightarrow \tau_{2}}$ is erased to a $\lambda^{\mathrm{u}}$ definition of the $\mathrm{Z}$ combinator $f i x$.

$$
\begin{aligned}
& \text { protect }_{\text {Unit }} \stackrel{\text { def }}{=} \lambda x \cdot x \quad \text { protect }_{\text {Bool }} \stackrel{\text { def }}{=} \lambda x \cdot x \\
& \operatorname{protect}_{\tau_{1} \times \tau_{2}} \stackrel{\text { def }}{=} \lambda \mathrm{y} \text {. }\left\langle\operatorname{protect}_{\tau_{1}} \mathrm{y} .1, \operatorname{protect}_{\tau_{2}} \mathrm{y} .2\right\rangle \\
& \operatorname{protect}_{\tau_{1} \uplus \tau_{2}} \stackrel{\text { def }}{=} \lambda y \text {. case } y \text { of } \mid \begin{array}{l}
\operatorname{inl} \mathrm{x} \mapsto \operatorname{inl}\left(\operatorname{protect}_{\tau_{1}} \mathrm{x}\right) \\
\operatorname{inr} \mathrm{x} \mapsto \operatorname{inr}\left(\operatorname{protect}_{\tau_{2}} \mathrm{x}\right)
\end{array} \\
& \operatorname{protect}_{\tau_{1} \rightarrow \tau_{2}} \stackrel{\text { def }}{=} \lambda \mathrm{y} \cdot \lambda \mathrm{x} \cdot \operatorname{protect}_{\tau_{2}}\left(\mathrm{y}\left(\text { confine }_{\tau_{1}} \mathrm{x}\right)\right) \\
& \text { confine }_{\text {Unit }} \stackrel{\text { def }}{=} \lambda y \text {. ( } y \text {; unit) } \\
& \text { confine }_{\text {Bool }} \stackrel{\text { def }}{=} \lambda y \text {. if } y \text { then true else false } \\
& \text { confine }_{\tau_{1} \times \tau_{2}} \stackrel{\text { def }}{=} \lambda \mathrm{y} \text {. }\left\langle\text { confine }_{\tau_{1}} \mathrm{y} .1, \text { confine }_{\tau_{2}} \mathrm{y} .2\right\rangle \\
& \text { confine }_{\tau_{1} \uplus \tau_{2}} \stackrel{\text { def }}{=} \lambda y \text {. case } y \text { of } \mid \begin{array}{l}
\operatorname{inl} x \mapsto \operatorname{inl}\left(\text { confine }_{\tau_{1}} x\right) \\
\operatorname{inr} x \mapsto \operatorname{inr}\left(\text { confine }_{\tau_{2}} x\right)
\end{array} \\
& \text { confine }_{\tau_{1} \rightarrow \tau_{2}} \stackrel{\text { def }}{=} \lambda \mathrm{y} . \lambda \mathrm{x} \text {. confine }{\overline{\tau_{2}}}\left(\mathrm{y}\left(\operatorname{protect}_{\tau_{1}} \mathrm{x}\right)\right)
\end{aligned}
$$

Figure 9. Dynamic type checking wrappers: the second pass of the compiler.

The erase function can be considered as a compiler, but it is only a correct compiler, not a fully-abstract one, as explained in Example 4.1.

Example 4.1 (Erasure is correct but not secure [Patrignani et al., 2015, Fournet et al., 2013]). Consider the following, contextually equivalent $\lambda^{\tau}$ functions of type Unit $\rightarrow$ Unit:

$$
\lambda \mathbf{x}: \text { Unit. } \mathbf{x} \simeq \simeq_{c t x} \quad \lambda \mathbf{x}: \text { Unit. unit }
$$

The erase function will map these to the following $\lambda^{\mathrm{u}}$ functions:

$$
\lambda \mathrm{x} \cdot \mathrm{x} \quad \neq_{c t x} \quad \lambda \mathrm{x} . \text { unit }
$$

The results of erase are not contextually equivalent, essentially because applying them to a non-unit value like true will produce true for the left lambda and unit for the right lambda. In this example, contextual equivalence is not preserved because the original functions are only defined for Unit values, but their compilations can be applied to other values too.

Lemma 2 states that every $\lambda^{\tau}$ term is related to its erased term at its type.

Lemma 2 (Erase is semantics-preserving (for terms)). If $\Gamma \vdash \mathrm{t}: \tau$, then $\Gamma \vdash \mathrm{t} \square$ erase $(\mathrm{t}): \tau$.

An analogous result applies to program contexts:

Lemma 3 (Erase is semantics-preserving (for context)). For all $\mathfrak{C}$, if $\vdash \mathfrak{C}: \Gamma^{\prime}, \tau^{\prime} \rightarrow \Gamma$, then $\vdash \mathfrak{C} \square$ erase $(\mathfrak{C}): \Gamma^{\prime}, \tau^{\prime} \rightarrow \Gamma, \tau$.

One should intuitively understand this result as " $t$ behaves the same as erase(t) when both are treated as values of type $\tau$ ". The result does not specify what happens when we treat $t$ as a value of a different type, like we did in Example 4.1 to demonstrate a full abstraction failure. Intuitively, it only specifies a kind of equivalence reflection for the erase function, not preservation.

Remember that a fully-abstract compiler must protect terms from being used in ways that are not allowed by their type, as in Example 4.1. This is taken care of by the second pass of the compiler.

We construct a family of dynamic typechecking wrappers protect $_{\tau}$ and confine . protect $_{\tau}$ is a $\lambda^{\mathrm{u}}$ lambda term that wraps an argument to enforce that it can only be used in ways that are valid according to type $\tau$, as often done in secure compilation work [Patrignani et al., 2015, Bowman and Ahmed, 2015, Fournet et al., 2013, Ahmed and Blume, 2008]. Dually, confine ${ }_{\tau}$ wraps its argu- 
ment so that it can only behave in ways that are valid according to type $\tau$. In the definition, the cases for product and coproduct types simply recursively descend on their subterms preserving the expected syntax of a product or coproduct argument. Protecting at a function type means wrapping the function to confine its arguments and protect its results, and dually for confining at a function type. Finally, protecting at a base type (i.e., Unit or Bool) does nothing, simply because there is nothing one can do to a base value that is not allowed by its type. Confining a value at a base type is more interesting. Both for Unit and Bool values, we use the value in such a way that will only work when the value is actually of the correct type. If it is, we return the original value, otherwise the term will reduce to wrong. ${ }^{2}$

Example 4.2 (Protect and confine make a term secure). Consider the protect wrapper for type Unit $\rightarrow$ Unit protect Unit $\rightarrow$ Unit $_{\text {, }}$ which is (roughly) equal to $\lambda y . \lambda x \cdot y(x ;$ unit). Applying that

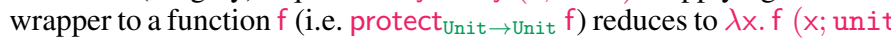
Applying this value to a non-unit value will simply evaluate to wrong, therefore addressing the issues of Example 4.1.

For the second pass of the compiler, Lemma 4 holds.

Lemma 4 (Protect and confine are semantics-preserving). If $\Gamma \vdash$ $\mathrm{t} \square_{\mathrm{n}} \mathrm{t}: \tau$ then $\Gamma \vdash \mathrm{t} \square_{\mathrm{n}}$ protect $_{\tau} \mathrm{t}: \tau$ and $\Gamma \vdash \mathrm{t} \square_{\mathrm{n}}$ confine $_{\tau} \mathrm{t}:$ $\tau$.

Lemma 4 states that if $\mathrm{t}$ is related to $\mathrm{t}$ at type $\tau$, then adding a protect $\tau$ or confine $\tau$ wrapper around $t$ does not change that. In other words, the wrappers do not change the behaviour of $t$ as long as they are treated as values of type $\tau$. In Section 5.5, we will have more to say about the security of the wrappers.

This section concludes with the definition of the compiler used in this paper.

Definition 1 (The $\llbracket \cdot \rrbracket$ compiler). If $\Gamma \vdash \mathrm{t}: \tau$, then $\mathrm{t}$ is compiled to $\llbracket \mathrm{t} \rrbracket$ and: $\llbracket \mathrm{t} \rrbracket \stackrel{\text { def }}{=} \operatorname{protect}_{\tau}(\operatorname{erase}(\mathrm{t}))$.

Lemmas 2 and 4 about the first and second pass of the compiler can be combined into Lemma 5 to obtain that a $\lambda^{\tau}$ term of type $\tau$ behaves like its compilation when both are treated as terms of type $\tau$.

Lemma $5(\llbracket \cdot \rrbracket$ is semantics-preserving). For all $\mathrm{t}$, if $\Gamma \vdash \mathrm{t}: \tau$ then $\Gamma \vdash \mathrm{t} \square \llbracket \mathrm{t} \rrbracket: \tau$.

\section{Approximate Back-Translation}

This section presents the core idea of our proof technique: the approximate back-translation. As explained in Section 1, the idea is to translate a target language program context $\mathfrak{C}$ to a source language program context $\langle\langle\mathfrak{C}\rangle\rangle_{n}$ which conservatively $n$-approximates $\mathfrak{C}$. Intuitively, this means that $\langle\langle\mathfrak{C}\rangle\rangle_{\mathrm{n}}$ behaves like $\mathfrak{C}$ for up to $n$ steps but it may diverge in cases where the original did not if $\mathfrak{C}$ takes more than $n$ steps. We will make this more precise in Section 5.2.

At the core of the approximate back-translation is the $\lambda^{\tau}$ type $\mathrm{UVal}_{\mathrm{n}}$. The type is essentially a $\lambda^{\tau}$ encoding of the unitype of $\lambda^{\mathrm{u}}$. Where the untyped context $\mathfrak{C}^{\mathfrak{C}}$ manipulates arbitrary $\lambda^{\mathrm{u}}$ values, its emulation $\langle\langle\mathfrak{C}\rangle\rangle_{n}$ manipulates values of type $U V$ al $l_{n}$. Section 5.1 defines $\mathrm{UVal}_{n}$ and the basic tools (constructors and destructors) for working with it. To explain how values in $U V$ a $l_{n}$ model values in $\lambda^{u}$, Section 5.2 fills in the missing piece of the logical relations of Fig. 7 by defining $\mathcal{V} \llbracket$ EmulDV $_{\mathrm{n} ; \mathrm{p}} \rrbracket_{\square}$.

The type $\mathrm{UVal}_{n}$ is sufficiently large to contain $n$-approximations of $\lambda^{u}$ values. However, it also contains approximations of $\lambda^{u}$ values up to less than n steps. This is crucial, as for a term to be well-typed

\footnotetext{
${ }^{2}$ Note that it would also be valid to produce a diverging term in this case, if $\lambda^{\mathrm{u}}$ had some form of dynamic type test which allowed us to do that.
}

the accuracy of the approximation can be less than $n$. In these cases values of type $\mathrm{UVal}_{\mathrm{n}}$ will be downgraded to a type $\mathrm{UVal}_{\mathrm{m}}$ with $m<n$. Dually, there will be cases where some values need to upgrade. Section 5.3 defines functions to perform value upgrading and downgrading.

With the definition of upgrading and downgrading, we have defined all the machinery that revolves around $\mathrm{UVal}_{n}$. Section 5.4 constructs the function emulate ${ }_{\mathrm{n}}$, responsible for emulating a context such that it translates a $\lambda^{u}$ term $t$ into a $\lambda^{\tau}$ term of type $U V_{a l}$. This function is easily extended to work with program contexts, producing contexts with hole of type $\mathrm{UVal}_{n}$ as expected.

However, remember from Fig. 3 in Section 1 that the goal of the back-translation is generating a context $\left\langle\left\langle\mathfrak{C}^{2}\right\rangle\right\rangle_{n}$ whose hole can be filled with $\lambda^{\tau}$ terms $t_{1}$ and $t_{2}$. However, the type of $t_{1}$ and $\mathrm{t}_{2}$ is not $\mathrm{UVal}_{\mathrm{n}}$ but an arbitrary $\lambda^{\tau}$ type $\tau$. Thus, there is a type mismatch between the hole of the emulated context emulate $\mathfrak{n}_{n}\left(\mathfrak{C}^{\mathfrak{c}}\right)$ and the terms that we want to plug in there. Since the emulated . contexts work with $U V_{a l}$ values, we need a function that wraps terms of an arbitrary type $\tau$ into a value of type $U V a l_{n}$. This is precisely what Section 5.5 defines, namely a function inject $t_{\tau ; \mathrm{n}}$ of type $\tau \rightarrow$ UValn $_{\text {. }}$

Finally, Section 5.6 defines the approximate back-translation function $\langle\langle\cdot\rangle\rangle_{\tau ; n}$, mapping a $\lambda^{u}$ context $\mathfrak{C}$ to a $\lambda^{\tau}$ context $\langle\langle\mathfrak{C}\rangle\rangle_{\tau ; n}$. The additional index $\tau$ w.r.t. earlier discussions is needed to introduce an appropriate call to inject $t_{\tau ; n}$ as discussed above, so that the hole of $\left\langle\left\langle\mathfrak{C}^{\mathfrak{C}}\right\rangle\right\rangle_{\tau ; n}$ is of type $\tau$. Plugging a term $\mathrm{t}_{1}$ in $\langle\langle\mathfrak{C}\rangle\rangle_{\tau ; n} n$ approximates plugging in the compilation $\llbracket \mathrm{t}_{1} \rrbracket$ in context $\mathfrak{C}$.

Right after the definition of each of the concepts discussed

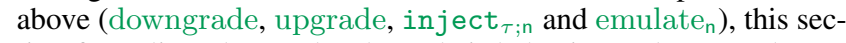
tion formalises the results about their behaviour. These results are expressed in terms of the logical relations of Fig. 7 and of the EmulDV $n ; p$ pseudo-type; they will be used to prove equivalence preservation in Section 6.

\subsection{UVal and its Tools}

The family of types $\mathrm{UVal}_{\mathrm{n}}$ is defined as follows:

$$
\begin{aligned}
& \mathrm{UVal}_{0} \stackrel{\text { def }}{=} \text { Unit } \\
& \mathrm{UVal}_{n+1} \stackrel{\text { def }}{=} \begin{array}{l}
\text { Unit } \uplus \mathrm{Unit}_{\mathrm{N}} \uplus \mathrm{Bool} \uplus\left(\mathrm{UVal}_{n} \times \mathrm{UVal}_{n}\right) \uplus \\
\left(\mathrm{UVal}_{n} \uplus \mathrm{UVal}_{n}\right) \uplus\left(\mathrm{UVal}_{n} \rightarrow \mathrm{UVal}_{n}\right)
\end{array}
\end{aligned}
$$

$\mathrm{UVal}_{\mathrm{n}}$ is the type that emulated $\lambda^{\mathrm{u}}$ terms have when backtranslated into $\lambda^{\tau}$. For every $n, \mathrm{UVal}_{n}$ is clearly a valid $\lambda^{\tau}$ type. At non-zero levels, the type $\mathrm{UVal}_{\mathrm{n}+1}$ is a disjunct sum of base values (the second occurrence of Unit and Bool), products and coproducts of $\mathrm{UVal}_{n} \mathrm{~s}$ and functions mapping a $\mathrm{UVal}_{n}$ to a $\mathrm{UVal}_{n}$. All of these cases are used to emulate a corresponding $\lambda^{u}$ value. Additionally, at every level including $n=0$, the type $U V_{a l}$ contains a Unit case which is needed to represent an arbitrary $\lambda^{\mathrm{u}}$ value in cases where the precision of the approximate emulation is insufficient to provide more information. Note that the two occurrences of Unit in the definition of $\mathrm{UVal}_{\mathrm{n}+1}$ are intentional. The first is used for imprecisely representing arbitrary $\lambda^{\mathrm{u}}$ terms while the second accurately represents $\lambda^{\mathrm{u}}$ unit values.

To work with $\mathrm{UVal}_{n}$ values, we need basic tools for dealing with sum types: tag injections and case extractions (Fig. 10). Func-

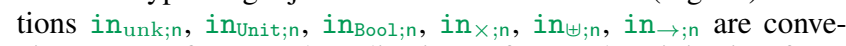
nient names for nested applications of coproduct injection functions for the nested coproduct in the definition of $\mathrm{UVal}_{n+1}$. The term $u_{n} k_{n}$ produces either the single value of $\mathrm{UVal}_{0}$ or uses $i n_{u n k ; n}$ to produce a $\mathrm{UVal}_{n+1}$ value representing a 0 -precision approximate back-translation of an arbitrary untyped term. For using $\mathrm{UVal}_{n}$ values, we define functions case $\mathrm{Unit;n}_{\mathrm{n}}$, case $_{\mathrm{B} \circ o 1 ; \mathrm{n}}, \mathrm{case}_{\times ; \mathrm{n}}$, case $_{\uplus ; n}$, case $\rightarrow ; n$ using a somewhat liberal pattern matching syntax that can be easily desugared to nested case expressions. The 


$$
\begin{aligned}
& \text { in }_{\text {unk;n }}: \mathrm{UVal}_{\mathrm{n}+1} \\
& \text { in Unit;n }_{\text {Unit } \rightarrow \mathrm{UVal}_{\mathrm{n}+1}} \\
& \text { in }_{\mathrm{Bool} ; \mathrm{n}}: \mathrm{Bool} \rightarrow \mathrm{UVal}_{\mathrm{n}+1} \\
& \text { in }_{\times ; n}:\left(\mathrm{UVal}_{n} \times \mathrm{UVal}_{\mathrm{n}}\right) \rightarrow \mathrm{UVal}_{\mathrm{n}+1} \\
& \text { in }_{\uplus ; n}:\left(\mathrm{UVal}_{n} \uplus \mathrm{UVal}_{\mathrm{n}}\right) \rightarrow \mathrm{UVal}_{\mathrm{n}+1} \\
& \text { in } \rightarrow ; n:\left(\mathrm{UVal}_{\mathrm{n}} \rightarrow \mathrm{UVal}_{\mathrm{n}}\right) \rightarrow \mathrm{UVal}_{\mathrm{n}+1} \\
& \text { omega }_{\tau}: \tau \quad \text { omega }_{\tau} \stackrel{\text { def }}{=} \text { fix Unit } \rightarrow \tau_{\tau}(\lambda \mathbf{x}: \text { Unit } \rightarrow \tau \text {. } \mathbf{x}) \text { unit } \\
& \text { case Unit;n }_{\text {UVal }} \text { UV }_{n+1} \rightarrow \text { Unit } \quad \text { case }_{\text {Bool;n }}: \text { UVal }_{n+1} \rightarrow \text { Bool } \\
& \text { case }_{\times ; n}: \mathrm{UVal}_{n+1} \rightarrow\left(\mathrm{UVal}_{\mathrm{n}} \times \mathrm{UVal}_{\mathrm{n}}\right) \\
& \text { case }_{\uplus ; \mathrm{n}}: \mathrm{UVal}_{\mathrm{n}+1} \rightarrow\left(\mathrm{UVal}_{\mathrm{n}} \uplus \mathrm{UVal}_{\mathrm{n}}\right) \\
& \text { case } \rightarrow ; n_{n}: \mathrm{UVal}_{\mathrm{n}+1} \rightarrow \mathrm{UVal}_{\mathrm{n}} \rightarrow \mathrm{UVal}_{\mathrm{n}} \\
& \text { case Unit;n }^{\text {def }}=\lambda \mathbf{x}: \mathrm{UVal}_{\mathrm{n}+1} \text {. case } \mathbf{x} \text { of }\left\{\text { in }_{\text {Unit } ; n} \mathrm{x} \mapsto \mathbf{x} ;{ }_{-} \mapsto \text { omega }\right\} \\
& \text { case }_{\text {Bool; }} \stackrel{\text { def }}{=} \lambda \mathbf{x}: \mathrm{UVal}_{\mathrm{n}+1} \text {. case } \mathbf{x} \text { of }\left\{\text { in }_{\mathrm{Bool} ; \mathrm{n}} \mathrm{x} \mapsto \mathbf{x} ;_{-} \mapsto \text { omega }\right\} \\
& \text { case }_{x ; n} \stackrel{\text { def }}{=} \lambda \mathbf{x}: \mathrm{UVal}_{\mathrm{n}+1} \text {. case } \mathbf{x} \text { of }\left\{\text { in }_{\mathrm{X} ; \mathrm{n}} \mathrm{x} \mapsto \mathbf{x} ;{ }_{-} \mapsto \text { omega }\right\} \\
& \text { case } \uplus ; \mathrm{n}_{\text {def }}^{=} \lambda \mathbf{x}: \mathrm{UVal}_{\mathrm{n}+1} \text {. case } \mathbf{x} \text { of }\left\{\text { in }_{\uplus ; n} \mathbf{x} \mapsto \mathbf{x} ;_{-} \mapsto \text { omega }\right\} \\
& \text { case } \rightarrow ; \mathrm{n}_{\text {def }}^{=} \lambda \mathbf{x}: \mathrm{UVal}_{\mathrm{n}+1} \cdot \lambda \mathbf{y}: \mathrm{UVal}_{\mathrm{n}} \text {. case } \mathbf{x} \text { of }
\end{aligned}
$$

Figure 10. Basic tools for working with $\mathrm{UVal}_{n}$. The subscript of omega is omitted when it is clear from the context.

functions are lambdas that inspect their $\mathrm{UVal}_{\mathrm{n}+1}$ argument and return the contained value if it is in the appropriate branch of the coproduct, or diverge otherwise. To achieve divergence, we use a term omega $\tau$ constructed using fix. We simply write omega when the type $\tau$ can be inferred from the context.

\section{$5.2 \lambda^{\mathrm{u}}$ Values vs. UVal}

To make the correspondence between a $\lambda^{\mathrm{u}}$ term and its emulation in $U V a l_{n}$ more exact, this section fills in the definition of $\mathcal{V} \llbracket E m u l D V_{n ; p} \rrbracket_{\square}$, the missing piece of the logical relations of Fig. 7. Recall that the intuition is that EmulDV $n$;p is a token type that is used to relate $\lambda^{u}$ terms to their $\lambda^{\tau}$ emulation of type UValn. This relation is done up to an approximateness degree, denoted with $p::=$ precise | imprecise, that is explained below. Intuitively, the previously presented cases of the logical relations define the relation between a $\lambda^{\tau}$ term and its compilation. The $\mathcal{V} \llbracket E_{\text {EmulDV }} ; \mathrm{p} \rrbracket_{\square}$ case defines the relation between a $\lambda^{\mathrm{u}}$ term and its UValn-typed back-translation, as motivated in Example 5.1.

Example 5.1 (The need of EmulDV). Consider the term $\mathrm{t} \equiv$ in $_{\text {Bool;1 }}$ true. Since UVal $_{n}$ is a sum type, according to the definition of $\mathcal{V} \llbracket \tau \uplus \tau^{\prime} \rrbracket$, it can be related only to terms that have the same tag. However, for the back-translation we do not want this, we want that term to be related to the $t$ term that $t$ approximates (in this case, true).

Type EmulDV $V_{n ; p}$ serves the purpose of bridging the syntactic difference, allowing $i n_{\mathrm{B} \circ \mathrm{O}} 1$ true and true to be related.

Before explaining the definition of the logical relations for $E m u l D V_{n ; p}$, we should explain how we elaborate on the approximateness of the correspondence.

Example 5.2. Consider the $\mathrm{UVal}_{6}$ value

$$
\text { in }_{\times ; 5}\left\langle\text { in }_{\uplus ; 4}\left(\text { inl unk } k_{4}\right), \text { unk }_{5}\right\rangle
$$

This value might be used by the approximate back-translation to represent the $\lambda^{\mathrm{u}}$ term $\langle$ inl $\langle$ unit, true $\rangle, \lambda x . x\rangle$. Our $\mathcal{V} \llbracket E_{\text {mulDV }} ; p \rrbracket \square$ specification will enforce that terms of the form $\operatorname{in}_{\times ; n}\langle\cdot, \cdot\rangle$ or $\operatorname{in}_{\uplus ; n}$ (inl · ) represent the corresponding $\lambda^{\mathrm{u}}$ constructs, but terms unk $_{4}$ and $u k_{5}$ can represent arbitrary terms (in this case: a pair of base values and a lambda).

The limited size of the type $U \mathrm{Val}_{n}$ sometimes forces us to resort to $u_{k} k_{n}$ values in the back-translation, making it approximate. However, $\mathcal{V} \llbracket E m u I D V_{n ; p} \rrbracket_{\square}$ does not allow these $u_{n} k_{n}$ values to occur anywhere, because they could compromise the required precision of our approximate back-translation.

In fact, $\mathcal{V} \llbracket E m u l D V_{n ; p} \rrbracket_{\square}$ provides two different specifications for the occurrence of $u_{\mathrm{n}} \mathrm{k}_{\mathrm{n}}$, depending on the value of $p$. The case where $p=$ imprecise is used when we are proving $\langle\langle\mathfrak{C}\rangle\rangle_{n} \lesssim \mathfrak{C}$, which means roughly that termination of $\langle\langle\mathfrak{C}\rangle\rangle_{n}$ in any number of steps implies termination of $\mathfrak{C}$. In this case, $\mathcal{V} \llbracket \mathrm{EmulDV}_{\mathrm{n} ; \mathrm{p}} \rrbracket_{\square}$ allows unk $_{\mathrm{n}}$ values to occur everywhere in a back-translation term, and they can correspond to arbitrary $\lambda^{u}$ terms. These mild requirements on the correspondence of $\lambda^{\mathrm{u}}$ terms place a large burden on the code in a back-translation $\langle\langle\mathfrak{C}\rangle\rangle_{n}$. This code must be able to deal with $u_{n} k_{n}$ values and produce behaviour for them that approximates the behaviour of $\mathfrak{C}$ for the arbitrary values that the $u_{n} k_{n} s$ correspond with. Luckily, when we are proving $\langle\langle\mathfrak{C}\rangle\rangle_{n} \lesssim \mathfrak{C}$, we can achieve this by simply making all the functions in our back-translation diverge whenever they try to use a $U V a_{n}$ value that happens to be an $u_{k}$. This is sufficient because the approximation $\langle\langle\mathfrak{C}\rangle\rangle_{n} \lesssim \mathfrak{C}$ trivially holds when $\langle\langle\mathfrak{C}\rangle\rangle_{n}$ diverges: it essentially only requires that $\mathfrak{C}$ terminates whenever $\langle\langle\mathfrak{C}\rangle\rangle_{n}$ does, but nothing needs to be shown when the latter diverges.

Example 5.3 (Relatedness with imprecise). Consider the term $\mathrm{t} \equiv$ in $_{\times ; 42}\left\langle\right.$ unk $_{42}$, unk $\left.k_{42}\right\rangle$. This term will be related to $\left\langle\mathrm{t}_{1}, \mathrm{t}_{2}\right\rangle$ at pseudo-type EmulDV 43 ;imprecise for any terms $t_{1}$ and $t_{2}$ and in any world.

The increased index 43 is needed because $t$ is tagged at 42 , so we need an additional step to unfold the tagging.

The case when $p=$ precise specifies where values $u n k_{n}$ are allowed when we are proving that $\langle\langle\mathfrak{C}\rangle\rangle_{n} \gtrsim_{n} \mathfrak{C}$, meaning roughly that termination of $\mathfrak{C}$ in less than $n$ steps implies termination of $\langle\langle\mathfrak{C}\rangle\rangle_{n}$. In this case, the requirements on the back-translation correspondence are significantly stronger: $u n k_{n}$ is simply ruled out by the definition of $\mathcal{V} \llbracket E m u l D V_{n ; p} \rrbracket_{\square}$. That does not mean, however, that $u_{n} k_{n}$ cannot occur inside related terms, rather that $u n k_{n}$ can only occur at depths that cannot be reached using the number of steps in the world.

Example 5.4 (Relatedness with precise). Consider again the term $\mathrm{t} \stackrel{\text { def }}{=}$ in $_{\times ; 42}\left\langle u_{n k_{42}}, u_{n k}\right\rangle$. This term will still be related by EmulDV 43 ; precise to $t \stackrel{\text { def }}{=}\left\langle t_{1}, t_{2}\right\rangle$ for any terms $t_{1}$ and $t_{2}$, but only in worlds $\underline{W}$ such that lev $(\underline{\mathrm{W}})=0$. More precisely, our specification will state that $(\underline{\mathrm{W}}, \mathrm{t}, \mathrm{t}) \in \mathcal{V} \llbracket E_{\mathrm{EmulDV}}$ 43; precise $\rrbracket_{\square}$ iff

$\left(\underline{\mathrm{W}},\left\langle\mathrm{unk}_{42}, \mathrm{unk}_{42}\right\rangle, \mathrm{t}\right) \in \mathcal{V} \llbracket \mathrm{EmulDV}_{42 ; \text { precise }} \times \mathrm{EmulDV}_{42 ; \text { precise }} \rrbracket \square$

By the definition in Fig. 7 , this requires in turn that $\left(\underline{W}, u k_{42}, t_{1}\right)$ and $\left(\underline{W}, u_{n} k_{42}, t_{2}\right)$ are in $\triangleright \mathcal{V} \llbracket E_{m u l} V_{42, \text { precise }} \rrbracket_{\square}$. However if $\operatorname{lev}(\underline{\mathrm{W}})=0$, then this is true by definition of the $\triangleright$ operator, independent of the requirements of $\mathcal{V} \llbracket E_{\text {EmulDV }}$, precise $\rrbracket_{\square}$.

Intuitively, it is sufficient to only forbid $u_{n} k_{n}$ at depths lower than the number of steps left in the world because we are proving $\langle\langle\mathfrak{C}\rangle\rangle_{n} \gtrsim_{n} \mathfrak{C}$ (emphasis on the index ${ }_{n}$ of $\gtrsim_{n}$ ). So, if $\mathfrak{C}$ terminates in less than $n$ steps, then the evaluation of $\mathfrak{C}$ cannot have used values that are deeper than level $n$ in any $\mathrm{UVal}_{n}$. The corresponding execution of $\langle\langle\mathfrak{C}\rangle\rangle_{n}$ will also not have had a chance to encounter the $u_{n} k_{n} s$. Therefore, the executions must have behaved identically.

With this approximation aspect explained, Fig. 11 presents the definition of $\mathcal{V} \llbracket E m u l D V_{n ; p} \rrbracket_{\square}$. For relating terms $v$ and $v$ in a world $\underline{\mathrm{W}}$, the definition requires that $\mathrm{v}$ has the right type and that $p=$ imprecise if $\mathrm{v}$ is $u_{\mathrm{n}} \mathrm{n}$. Additionally, the structure of the $\lambda^{\tau}$ 


$$
\begin{aligned}
& \mathcal{V} \llbracket E_{m u l D V} ; \mathrm{p} \rrbracket_{\square} \stackrel{\text { def }}{=}\{(\underline{\mathrm{W}}, \mathrm{v}, \mathrm{v}) \mid \mathrm{v}=\text { unit and } p=\text { imprecise }\} \\
& \mathcal{V} \llbracket \mathrm{EmulDV}_{\mathrm{n}+1 ; \mathrm{p}} \rrbracket_{\square} \stackrel{\text { def }}{=}
\end{aligned}
$$

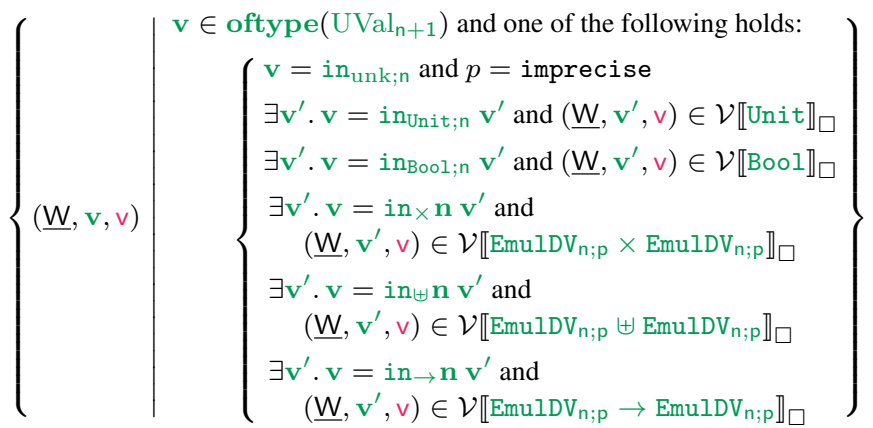

Figure 11. Specifying the relation between $\lambda^{u}$ values and their emulation in $\mathcal{V} \llbracket E m u l D V_{n ; p} \rrbracket_{\square}$.

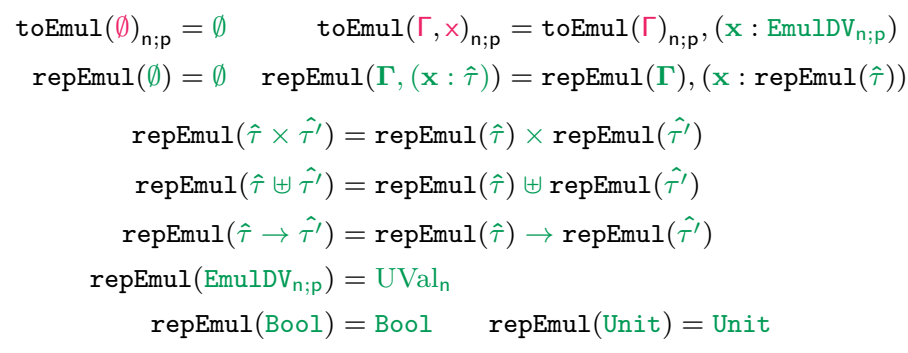

Figure 12. Helper functions for $E_{m u l D V} ; p$.

term stripped of its $\mathrm{UVal}_{n}$ tag and the structure of the $\lambda^{\mathrm{u}}$ term must coincide. Formally, this is expressed by the following conditions: $\left(\underline{W}, v^{\prime}, v\right)$ are in $\mathcal{V} \llbracket \mathcal{B} \rrbracket_{\square}, \mathcal{V} \llbracket E m u l D V_{n ; p} \times E m u l D V_{n ; p} \rrbracket_{\square}$,

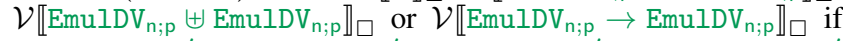
$\mathrm{v}=\operatorname{in}_{\mathcal{B} ; n} \mathbf{v}^{\prime}, \mathbf{v}=i n_{\times ; n} \mathbf{v}^{\prime}, \mathbf{v}=i n_{\uplus ; n} \mathbf{v}^{\prime}$ or $\mathbf{v}=i n_{\rightarrow ; n} \mathbf{v}^{\prime}$ respectively.

In addition to EmulDV $V_{n ; p}$, we still need to define two helper functions (Fig. 12) related to it. The first, repEmul(·), was left open in Fig. 7. It re-maps all variables of a $\Gamma$ that are of type EmulDV $n$ to type $\mathrm{UVal}_{n}$. A second function, toEmul $(\cdot)_{\mathrm{n} ; \mathrm{p}}$, turns an untyped $\Gamma$ into one where all variables are mapped to $E_{n, p}{ } D V_{n ; p}$.

The adequacy property of the logical relations (Lemma 1) hold for the complete definition of the logical relations, including the definition for $\mathcal{V} \llbracket E m u l D V_{n ; p} \rrbracket$.

\subsection{Upgrading and Downgrading Values}

Figure 13 defines the functions downgrade ${ }_{n ; d}: \mathrm{UVal}_{n+d} \rightarrow \mathrm{UVal}_{n}$ and upgrade $_{n ; d}: \mathrm{UVal}_{n} \rightarrow \mathrm{UVal}_{n+\mathrm{d}}$ (by induction on $n$ ) that we talked about before. Most cases simply work structurally over the type, but some are more interesting. There is a contravariance in the cases for function values in both downgrade $e_{n ; d}$ and upgrade $_{n ; d}$ : a function $\mathrm{UVal}_{n} \rightarrow \mathrm{UVal}_{n}$ is turned into a function of type $\mathrm{UVal}_{\mathrm{n}+\mathrm{d}} \rightarrow \mathrm{UVal}_{\mathrm{n}+\mathrm{d}}$ by constructing a wrapper that downgrades the argument and upgrades the result and vice versa. Unknown values are always mapped to unknown values, but additionally, the case for downgrade $n$ :d when $n=0$ will throw away the information contained in its argument of type $\mathrm{UVal}_{d}$ and simply returns the single unknown value in $\mathrm{UVal}_{0}$. Note that downgrade dod $_{\text {a }}$ and upgrade $_{n ; d}$ are not inverse functions, since downgrade ${ }_{n ; d}$ throws away information that was previously there. While $t \equiv$ downgrade $_{n ; d}$ upgrade $_{n ; d} t$, the reverse

$$
\begin{aligned}
& \text { downgrade }_{\mathrm{n} ; \mathrm{d}}: \mathrm{UVal}_{\mathrm{n}+\mathrm{d}} \rightarrow \mathrm{UVal}_{\mathrm{n}} \\
& \text { downgrade } 0 ; \mathrm{d} \stackrel{\text { def }}{=} \lambda \mathbf{v}: \mathrm{UVal}_{\mathrm{d}} \text {. } \mathrm{unk}_{0} \\
& \text { downgrade }_{n+1 ; d} \stackrel{\text { def }}{=} \lambda \mathbf{x}: \mathrm{UVal}_{n+d+1} \text {. case } \mathbf{x} \text { of } \\
& \text { in }_{\text {unk;n+d }} \mapsto \text { in }_{\text {unk;n }} \\
& \text { in Unit;n+d } \mathbf{y} \mapsto \text { inUnit;n } \mathbf{y} \\
& \text { in }_{\text {Bool;n+d }} \mathbf{y} \mapsto \text { in }_{\text {Bool; }} \mathbf{y} \\
& \text { in }_{\times ; n+d} \mathbf{y} \mapsto \text { in }_{\times ; n}\left\langle\text { downgrade }_{n ; d} \mathbf{y} . \mathbf{1} \text {, downgrade } \text { dowd }_{n} \mathbf{y} . \mathbf{2}\right\rangle \\
& \text { in }_{\uplus ; n+d} \mathbf{y} \mapsto \text { in }_{\uplus ; n} \text { case } \mathbf{y} \text { of } \mid \begin{array}{l}
\text { inl } \mathbf{x} \mapsto \operatorname{inl}\left(\text { downgrade }_{\mathrm{n} ; \mathrm{d}} \mathbf{x}\right) \\
\operatorname{inr} \mathbf{x} \mapsto \operatorname{inr}\left(\text { downgrade }_{\mathrm{n} ; \mathrm{d}} \mathbf{x}\right)
\end{array} \\
& \text { in } \rightarrow ; n+d \mathbf{y} \mapsto \text { in } \rightarrow ; n\left(\lambda \mathbf{z}: \text { UVal }_{n} \text {. downgrade } \text { dow }_{\text {id }}\right. \\
& \left.\left(\mathbf{y}\left(\text { upgrade }_{\mathrm{n} ; \mathrm{d}} \mathbf{z}\right)\right)\right)
\end{aligned}
$$$$
\operatorname{upgrade}_{\mathrm{n} ; \mathrm{d}}: \mathrm{UVal}_{\mathrm{n}} \rightarrow \mathrm{UVal}_{\mathrm{n}+\mathrm{d}}
$$$$
\text { upgrade }_{0 ; \mathrm{d}} \stackrel{\text { def }}{=} \lambda \mathbf{x}: \mathrm{UVal}_{0} \cdot \mathrm{unk}_{\mathrm{d}}
$$$$
\text { upgrade }_{n+1 ; d} \stackrel{\text { def }}{=} \lambda \mathbf{x}: \mathrm{UVal}_{\mathrm{n}+1} \text {. case } \mathbf{x} \text { of }
$$$$
\mathrm{in}_{\mathrm{unk} ; \mathrm{n}} \mapsto \text { in }_{\mathrm{unk} ; \mathrm{n}+\mathrm{d} ;}
$$$$
\text { in Unit;n } \mathbf{y} \mapsto \text { in }_{\text {Unit;n+d }} \mathbf{y} \text {; }
$$$$
\text { in }_{\text {Bool;n }} \mathbf{y} \mapsto \text { in }_{\text {Bool;n+d }} \mathbf{y} \text {; }
$$$$
\text { in }_{\times ; n} \mathbf{y} \mapsto \text { in }_{\times ; n+d}\left\langle\text { upgrade }_{n ; d} \mathbf{y} . \mathbf{1}, \text { upgrade }_{\mathrm{n} ; \mathrm{d}} \mathbf{y} . \mathbf{2}\right\rangle
$$$$
\text { in }_{\uplus ; \mathrm{n}} \mathbf{y} \mapsto \text { in }_{\uplus ; \mathrm{n}+\mathrm{d}} \text { case } y \text { of } \mid \begin{aligned}
& \text { inl } \mathbf{x} \mapsto \operatorname{inl}\left(\text { upgrade }_{\mathrm{n} ; \mathrm{d}} \mathbf{x}\right) \\
& \text { inr } \mathbf{x} \mapsto \operatorname{inr}\left(\operatorname{upgrade}_{\mathrm{n} ; \mathrm{d}} \mathbf{x}\right)
\end{aligned}
$$

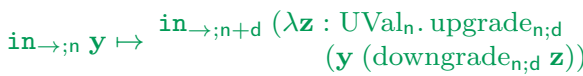

Figure 13. Upgrade and downgrade for $\mathrm{UVal}_{n}$.

( $t \equiv$ upgrade $_{n ; d}$ downgrade $\left._{n ; d} t\right)$ is not true, since applying downgrade first reduces precision.

Example 5.5 (Downgrading terms). Suppose that we want to emulate a $\lambda^{\mathrm{u}}$ term $\lambda \mathrm{x} .\langle\mathrm{x}, \mathrm{x}\rangle$ in $\mathrm{UVal}_{n}$ for a sufficiently-large $n$. We would expect roughly the following $\lambda^{\tau}$ term:

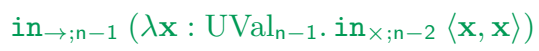

Indices $n-1$ and $n-2$ of the $\mathrm{UVal}_{n}$ constructors are imposed by the well-typedness constraints. However, even this is not enough to guarantee well-typedness. With a closer inspection, the variable $\mathrm{x}$ of type $\mathrm{UVal}_{n-1}$ is used where a term of type $\mathrm{UVal}_{n-2}$ is required (it is inside a pair tagged with in $_{\times ; n-2}$ ). This is a problem of type safety, not precision of approximation. Since $\mathrm{x}$ appears inside a pair, inspecting $\mathrm{x}$ for any number of steps requires at least one additional step to first project it out of the pair. In other words, for the pair to be a precise approximation up to $\leq n-1$ steps, $\mathrm{x}$ needs only to be precise up to $n-2$ steps. It is then safe to throw away one level of precision and downgrade $\mathrm{x}$ from type $\mathrm{UVal}_{\mathrm{n}-1}$ to $\mathrm{UVal}_{n-2}$.

We will use the function downgrade for the situation of Example 5.5 and similar ones in the next sections. In dual situations we will need to upgrade terms from type $\mathrm{UVal}_{n}$ to $U V \mathrm{Va} \mathrm{l}_{\mathrm{n}+\mathrm{d}}$. This will neither increase precision of the approximation, nor decrease it.

The correctness property for downgrade and upgrade is stated in the following lemma.

Lemma 6 (Compatibility lemma for upgrade $e_{n ; d}$ and downgrade $e_{n ; d}$ ). Suppose that either ( $n<m$ and $p=$ precise) or $(\square=\lesssim$ and $p=$ imprecise). Then

- If $\Gamma \vdash \mathrm{t} \square_{\mathrm{n}} \mathrm{t}:$ EmulDV $_{\mathrm{m}+\mathrm{d} ; \mathrm{p}}$, then $\Gamma \vdash$ downgrade $_{\mathrm{m} ; \mathrm{d}} \mathrm{t} \square_{\mathrm{n}}$ $t:$ EmulDV $m ; p$. 


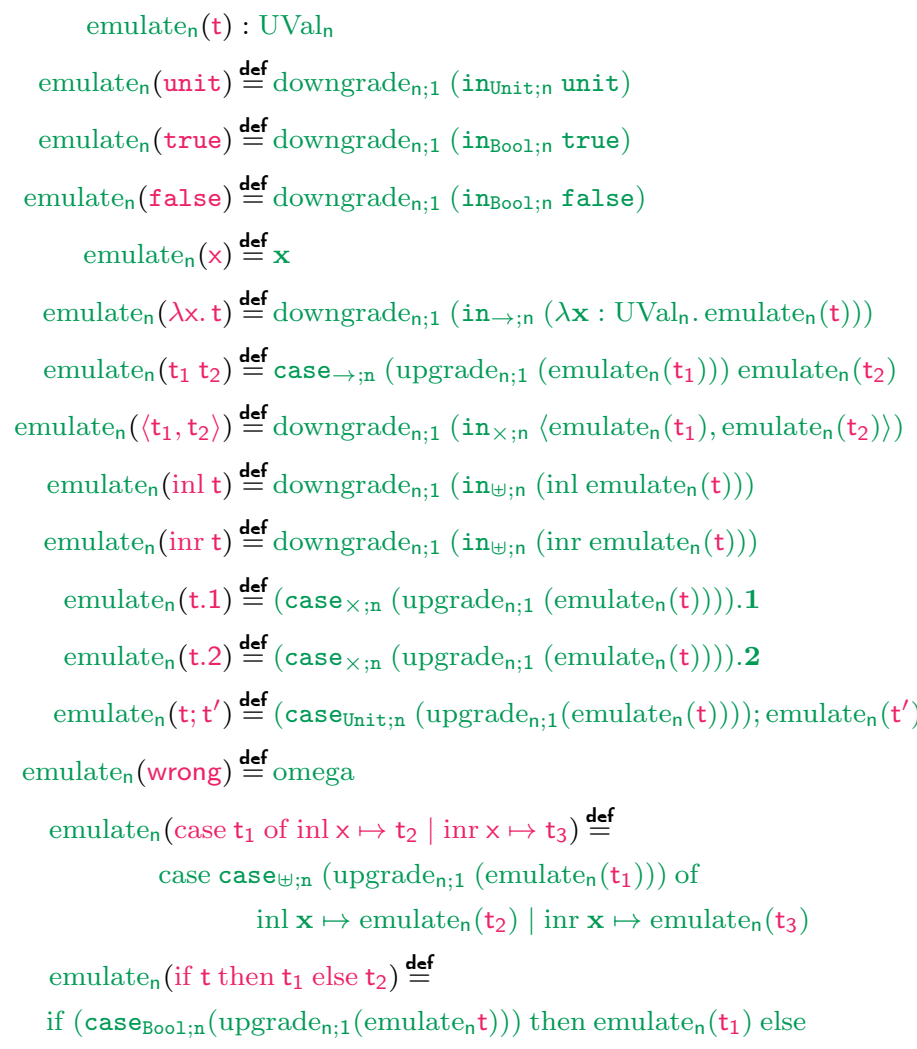

emulaten $\left(t_{2}\right.$

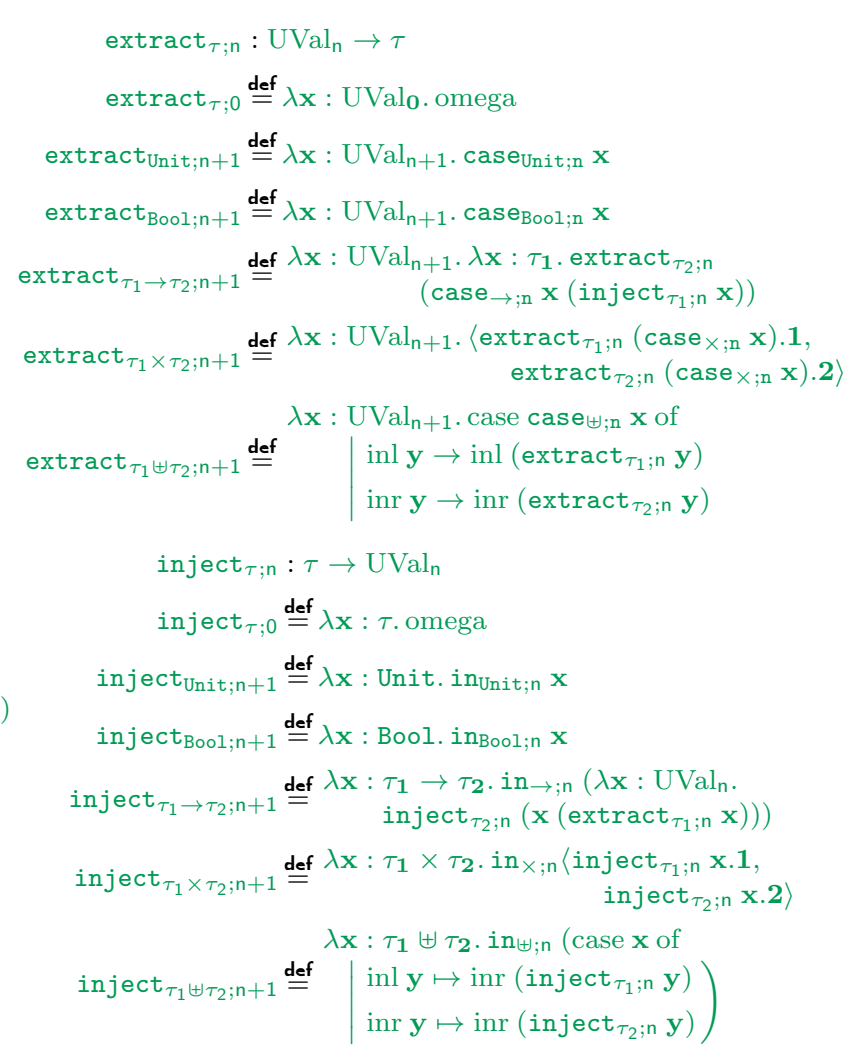

Figure 15. Injecting $\lambda^{\tau}$ values into $\mathrm{UVal}_{\mathrm{n}}$.

Lemma 7 (Emulate relates at EmulDV). If $\Gamma \vdash \mathrm{t}$, and if ( $m>n$ and $p=$ precise) or $(\square=\lesssim$ and $p=$ imprecise), then we have that toEmul $(\Gamma)_{\mathrm{m} ; \mathrm{p}} \vdash$ emulate $_{\mathrm{m}}(\mathrm{t}) \square_{\mathrm{n}} \mathrm{t}: \operatorname{EmulDV}_{\mathrm{m} ; \mathrm{p}}$.

Like Lemma 6, Lemma 7 requires that either $n<m$ (so that the results only hold in worlds $\mathrm{W}$ with $\operatorname{lev}(\mathrm{W}) \leq n<m)$, in which case $p=$ precise, or $\square=\lesssim$ and $p=$ imprecise. This again covers what we need for the two logical approximations of $\langle\langle\mathfrak{C}\rangle\rangle_{\mathrm{n}}$ in Fig. 3. The lemma states that the back-translation of any well-scoped term is related to the term by EmulDV $V_{m ; p}$, as intended.

An analogous result holds for contexts.

Lemma 8 (Emulate relates contexts at EmulDV). If $\vdash \mathfrak{C}: \Gamma^{\prime} \rightarrow \Gamma$, if ( $m>n$ and $p=$ precise) or $(\square=\lesssim$ and $p=$ imprecise $)$, then $\vdash$ emulate $_{\mathrm{m}}\left(\mathfrak{C}^{2}\right) \square_{\mathrm{n}} \mathfrak{C}^{\mathfrak{2}}: \operatorname{toEmul}\left(\Gamma^{\prime}\right)_{\mathrm{m} ; \mathrm{p}}$, EmulDV $_{\mathrm{m} ; \mathrm{p}} \rightarrow$ toEmul $(\Gamma)_{m ; p}, E_{m u l D V} ; p$

\subsection{Injection and Extraction of Terms}

One final thing is missing to construct a back-translation $\langle\langle\mathfrak{C}\rangle\rangle_{n}$ of an untyped program context $\mathfrak{C}$. While emulate $\mathfrak{n}_{n}(\mathfrak{C})$ produces a $\lambda^{\tau}$ context that expects a $\mathrm{UVal}_{n}$ value (just like $\mathfrak{C}^{\mathfrak{c}}$ expects an arbitrary $\lambda^{\mathrm{u}}$ value), the back-translation should accept values of a given type $\tau$ (the type of the terms $\mathbf{t}_{1}$ and $\mathbf{t}_{2}$ that we are compiling). To bridge this difference, Fig. 15 defines a $\lambda^{\tau}$ function inject $t_{\tau ; n}$ of type $\tau \rightarrow \mathrm{UVal}_{n}$ which injects values of an arbitrary type $\tau$ into $\mathrm{UVal}_{n}$. We define it mutually recursively with a dual function extract $_{\tau ; \mathrm{n}}: \mathrm{UVal}_{\mathrm{n}} \rightarrow \tau$ which is needed for contravariantly converting $\mathrm{UVal}_{n}$ arguments to the appropriate type in the inject $_{\tau ; \mathrm{n}}$ case for function types.

Generally, inject $\tau ;$;n converts a value $\mathrm{v}$ of type $\tau$ to a value of type $\mathrm{UVal}_{n}$ that behaves like the compilation $\llbracket \mathrm{v} \rrbracket$. The cases for base values use the related tagging and case (e.g., $i n_{\text {Unit;n }}$ and case $\left._{\text {Bool;n }}\right)$ to achieve this. For pair and sum values, inject $\tau$; n 
extract $_{\tau: \text { n }}$ simply recurse over the structure of the values, respectively applying in $_{\times ; n}, i_{\uplus ; n}$ and case $e_{\times ; n}$, case $_{\uplus ; n}$ to construct and destruct $U \mathrm{Val}_{n} \mathrm{~s}$ of a certain expected form. Note that when $U V \mathrm{Val} \mathrm{l}_{n}$ values do not have the form expected for type $\tau$, then extract $t_{\tau ; n}$ will diverge by definition of the case $\ldots ; n$ functions. This divergence corresponds to the wrong that we get when an untyped context attempts to use $\lambda^{\mathrm{u}}$ values as pairs, disjunct sum values or base values when those values are of a different form.

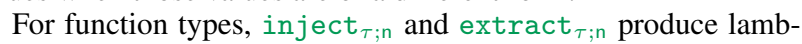
das that contravariantly extract and inject the argument and covariantly inject and extract the result. Finally, when $n=0$, then the size of our type is insufficient for extract $t_{\tau ; n}$ and inject inn $_{\tau, n}$ to accurately perform their intended function. Luckily, to obtain the necessary precision of our approximate back-translation, it is sufficient for them to simply diverge in this case: they simply return omega terms of the expected type.

For a value $\mathbf{v}$ of type $\tau$, inject $\tau$; n will produce a value $\mathrm{UVal}_{n}$ that behaves as the compilation of $\mathrm{v}, \llbracket \mathrm{v} \rrbracket$. More precisely and more generally, the following lemma states that if a term $t$ is related to a term $\mathrm{t}$ at type $\tau$ (intuitively if $\mathrm{t}$ behaves as $\mathrm{t}$ when used in a way that is valid according to type $\tau$ ), then inject $t_{\tau ; n}$ t behaves as the emulation of $\operatorname{protect}_{\tau} \mathrm{t}$. A dual result about extract $t_{\tau ; n}$ and confine $_{\tau}$ states (intuitively) that if a term $t$ behaves as an emulation of value $t$, then confine $e_{\tau} t$ will behave as extract $t_{\tau ; n} t$ when used in ways that are valid according to type $\tau$.

Lemma 9 (Inject is protect and extract is confine). If ( $m \geq n$ and $p=$ precise) or $(\square=\lesssim$ and $p=$ imprecise) and if $\hat{\Gamma} \vdash \mathrm{t} \square_{\mathrm{n}} \mathrm{t}: \tau$, then

$$
\hat{\Gamma} \vdash \text { inject }_{\tau ; \mathrm{m}} \mathrm{t} \square_{\mathrm{n}} \operatorname{protect}_{\tau} \mathrm{t}: \mathrm{EmulDV}_{\mathrm{m} ; \mathrm{p} .}
$$

If ( $m \geq n$ and $p=$ precise) or $(\square=\lesssim$ and $p=$ imprecise $)$ and if $\hat{\Gamma} \vdash \mathrm{t} \square_{\mathrm{n}} \mathrm{t}:$ EmulDV $\mathrm{m} ; \mathrm{p}$ then

$$
\hat{\Gamma} \vdash \operatorname{extract}_{\tau ; \mathrm{m}} \mathrm{t} \square_{\mathrm{n}} \text { confine }_{\tau} \mathrm{t}: \tau .
$$

Example 5.6. Consider again Example 4.1. We have that

$$
\emptyset \vdash \lambda \mathrm{x} \text { : Unit. } \mathrm{x} \square \lambda \mathrm{x} . \mathrm{x}: \text { Unit } \rightarrow \text { Unit. }
$$

$\lambda \mathbf{x}$ : Unit. $\mathbf{x}$ behaves like $\lambda \mathrm{x} . \mathrm{x}$, when the latter is used in ways that are valid for a value of type Unit $\rightarrow$ Unit. Lemma 9 then yields:

$$
\begin{aligned}
\emptyset \vdash \operatorname{inject}_{\tau ; n}(\lambda \mathbf{x}: & \text { Unit. } \mathbf{x}) \square_{\mathrm{n}} \\
& \operatorname{protect}_{\text {Unit } \rightarrow \text { Unit }}(\lambda \mathrm{x} . \mathrm{x}): \text { EmulDV }_{\mathrm{m} ; \mathrm{n}} .
\end{aligned}
$$

For $n$ sufficiently large and modulo some simplifications, these terms become:

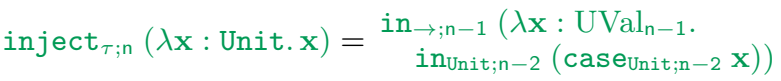

$$
\begin{aligned}
& \operatorname{protect}_{\text {Unit } \rightarrow \text { Unit }}(\lambda \mathrm{x} . \mathrm{x})=\lambda \mathrm{x} . \mathrm{x} \text {; unit }
\end{aligned}
$$

We invite the reader to verify that both expressions behave appropriately when applied to any values $\mathrm{v}$ and $\mathrm{v}$ that are related by EmulDV $_{n ; p}$ : for example ( $v=$ in $_{\text {Unit } ; n-1}$ unit and $v=$ unit), $\left(\mathrm{v}=\right.$ in $_{\rightarrow ; \mathrm{n}-1}\left(\lambda \mathrm{x}: \mathrm{UVal}_{\mathrm{n}-1} \cdot \mathrm{x}\right)$ and $\left.\mathrm{v}=\lambda \mathrm{x} \cdot \mathrm{x}\right)$ or $\left(\mathrm{v}=\mathrm{unk}_{\mathrm{n}}, \mathrm{v}\right.$ is any $\lambda^{\mathrm{u}}$ term and $\left.\square=\lesssim\right)$.

\subsection{Approximate Back-Translation}

We are now ready to define the approximate back-translation $\langle\langle\mathfrak{C}\rangle\rangle_{\tau ; n}$ of an arbitrary untyped context $\mathfrak{C}$ with a hole of type $\tau$. However, before we do, we need to correct a few simplifications that were made in Fig. 3.

First, as we have already explained, the back-translation $\langle\langle\mathfrak{C}\rangle\rangle_{n}$ does not just depend on $n$ but also on the type $\tau$ of the terms $t_{1}$ and $\mathrm{t}_{2}$ that we are compiling. As such, we define the back-translation with $\tau$ as an additional parameter.

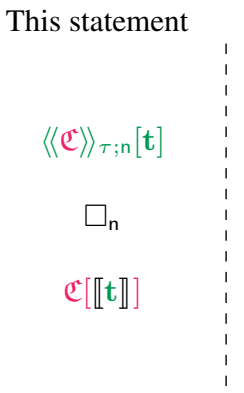

expands to this

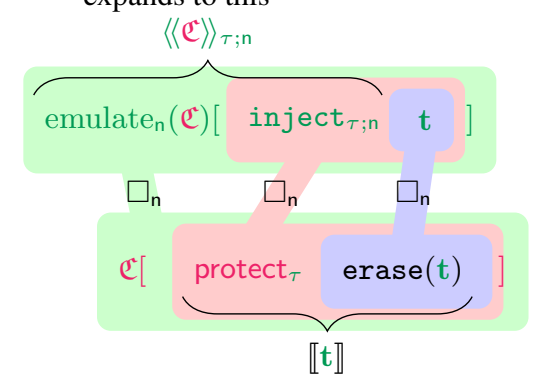

Terms related by Lemma 2

Terms related by Lemma 8

Terms related by Lemma 9

Figure 16. A more accurate picture of related components of compiled term $\mathrm{t}$, program context $\mathfrak{C}$, compilation $\llbracket \mathrm{t} \rrbracket$ and emulation $\langle\langle\mathfrak{C}\rangle\rangle_{\tau ; \mathrm{n}}$ than in the simplified Fig. 3.

Definition 2 (n-approximate back-translation $\left.\langle\langle\cdot\rangle\rangle_{\tau ; n}\right)$. The $n$ approximate back-translation of a context $\mathfrak{C}$ with a hole of type $\tau$ is defined as follows. $\langle\langle\mathfrak{C}\rangle\rangle_{\tau ; \mathrm{n}} \stackrel{\text { def }}{=}$ emulate $_{\mathrm{n}+1}(\mathfrak{C})\left[\right.$ inject $\left._{\tau ; \mathrm{n}} \cdot\right]$

A second simplification in Fig. 3 was the fact that we claimed $\langle\langle\mathfrak{C}\rangle\rangle_{n} \gtrsim_{n} \mathfrak{C}$ and $\langle\langle\mathfrak{C}\rangle\rangle_{n} \lesssim \mathfrak{C}$. Fig. 16 shows a more accurate picture of the relations that we have. As we will see in the next section, this more accurate picture still allows us to conclude the facts that $\emptyset \vdash\left\langle\left\langle\mathfrak{C}^{2}\right\rangle\right\rangle_{\tau ; n}\left[\mathrm{t}_{1}\right] \gtrsim_{\mathrm{n}} \mathfrak{C}\left[\llbracket \mathrm{t}_{1} \rrbracket\right]:$ EmulDV $V_{\mathrm{n} ; \text { precise }}$ and $\emptyset \vdash\left\langle\left\langle\mathfrak{C}^{2}\right\rangle\right\rangle_{\tau ; \mathrm{n}}\left[\mathrm{t}_{2}\right] \lesssim_{\mathrm{n}^{\prime}} \mathfrak{C}\left[\llbracket \mathrm{t}_{2} \rrbracket\right]:$ EmulDV $V_{\mathrm{n} ; \text { imprecise }}$ so that the proof goes through unchanged.

The correctness of $\langle\langle\cdot\rangle\rangle_{\tau ; \mathrm{n}}$ is captured in Lemma 10.

Lemma 10 (Correctness of $\left.\langle\langle\cdot\rangle\rangle_{\tau ; n}\right)$. If ( $m \geq n$ and $p=$ precise) or $\left(\square=\lesssim\right.$ and $p=$ imprecise), then $\Gamma \vdash \mathrm{t} \square_{\mathrm{n}} \mathrm{t}: \tau$ implies $\Gamma \vdash\langle\langle\mathfrak{C}\rangle\rangle_{\tau ; m}[\mathbf{t}] \square_{\mathrm{n}} \mathfrak{C}\left[\right.$ protect $\left._{\tau} \mathrm{t}\right]:$ EmulDV $_{\mathrm{m} ; \mathrm{p}}$.

Proof. Follows from Lemmas 8 and 9

\section{Compiler Full-Abstraction}

This section presents the proof that the compiler $\llbracket \cdot \rrbracket$ is fully-abstract (Theorem 3) in terms of the logical relations of Fig. 7. As previously mentioned, this results in proving equivalence reflection (Theorem 1) and preservation (Theorem 2). As suggested by Fig. 1 in Section 1, the lemmas presented in Section 4 are enough to prove equivalence reflection for $\llbracket \cdot \rrbracket$. Dually, as suggested by Fig. 3 in Section 1, the lemmas presented in Section 5 are enough to prove equivalence preservation for $\llbracket \cdot \rrbracket$.

Recall from Definition 1 that $\llbracket \mathrm{t} \rrbracket$ is $\operatorname{protect}_{\tau}(\operatorname{erase}(\mathrm{t}))$.

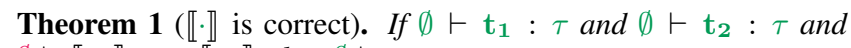
$\emptyset \vdash \llbracket \mathrm{t}_{1} \rrbracket \simeq_{c t x} \llbracket \mathrm{t}_{2} \rrbracket$, then $\emptyset \vdash \mathrm{t}_{1} \simeq_{c t x} \mathrm{t}_{2}: \tau$.

Proof. Take $\mathfrak{C}$ so that $\vdash \mathfrak{C}: \emptyset, \tau \rightarrow \emptyset, \tau^{\prime}$. By definition of $\simeq_{c t x}$, we need to prove that $\mathfrak{C}\left[\mathbf{t}_{1}\right] \Downarrow$ iff $\mathfrak{C}\left[\mathbf{t}_{2}\right]$. By symmetry, it suffices to prove the $\Rightarrow$ direction.

So, assume that $\mathfrak{C}\left[\mathbf{t}_{1}\right] \Downarrow$. We need to prove that $\mathfrak{C}\left[\mathbf{t}_{2}\right] \Downarrow$.

Define $\mathfrak{C} \stackrel{\text { def }}{=}$ erase $(\mathfrak{C})$, Lemma 3 yields $\vdash \mathfrak{C} \square \mathfrak{C}: \emptyset, \tau \rightarrow \emptyset, \tau^{\prime}$.

By Lemma 5, we get $\emptyset \vdash \mathrm{t}_{1} \square \llbracket \mathrm{t}_{1} \rrbracket: \tau$ and $\emptyset \vdash \mathrm{t}_{2} \square \llbracket \mathrm{t}_{2} \rrbracket: \tau$.

By definition of $\vdash \mathfrak{C} \square \mathfrak{C}: \emptyset, \tau \rightarrow \emptyset, \tau^{\prime}$, we get (specifically) that $\emptyset \vdash \mathfrak{C}\left[\mathrm{t}_{1}\right] \gtrsim \mathfrak{C}\left[\llbracket \mathrm{t}_{1} \rrbracket\right]: \tau^{\prime}$ and $\emptyset \vdash \mathfrak{C}\left[\mathrm{t}_{2}\right] \lesssim \mathfrak{C}\left[\llbracket \mathrm{t}_{2} \rrbracket\right]: \tau^{\prime}$.

$\mathfrak{C}\left[\mathrm{t}_{1}\right] \Downarrow$ and $\emptyset \vdash \mathfrak{C}\left[\mathrm{t}_{1}\right] \square \mathfrak{C}\left[\llbracket \mathrm{t}_{1} \rrbracket\right]: \tau^{\prime}$ imply $\mathfrak{C}^{c}\left[\llbracket \mathrm{t}_{1} \rrbracket\right] \Downarrow$ by Lemma 1. From $\llbracket \mathrm{t}_{1} \rrbracket \simeq_{c t x} \llbracket \mathrm{t}_{2} \rrbracket$ and $\mathfrak{C}\left[\llbracket \mathrm{t}_{1} \rrbracket\right] \Downarrow$, we get that $\mathfrak{C}\left[\llbracket \mathrm{t}_{2} \rrbracket\right] \Downarrow$. $\emptyset \vdash \mathfrak{C}\left[\mathrm{t}_{2}\right] \square \mathfrak{C}\left[\llbracket \mathrm{t}_{2} \rrbracket\right]: \tau^{\prime}$ and $\mathfrak{C}\left[\llbracket \mathrm{t}_{2} \rrbracket\right] \Downarrow$ yield $\mathfrak{C}\left[\mathrm{t}_{2}\right] \Downarrow$ by Lemma 1 . 


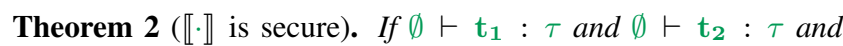
$\mathbf{t}_{1} \simeq_{c t x} \mathbf{t}_{2}: \tau$, then $\llbracket \mathrm{t}_{1} \rrbracket \simeq_{c t x} \llbracket \mathrm{t}_{2} \rrbracket$.

Proof. Note that $\operatorname{protect}_{\tau}\left(\operatorname{erase}\left(\mathrm{t}_{1}\right)\right)=\llbracket \mathrm{t}_{1} \rrbracket$ by definition and similarly for $\mathrm{t}_{2}$.

Take a $\vdash \mathfrak{C}: \emptyset \rightarrow \emptyset$ and suppose that $\mathfrak{C}\left[\operatorname{protect}_{\tau}\left(\operatorname{erase}\left(\mathrm{t}_{1}\right)\right)\right] \Downarrow$, then we need to show that $\mathfrak{C}\left[\operatorname{protect}_{\tau}\left(\operatorname{erase}\left(\mathrm{t}_{2}\right)\right)\right] \Downarrow$.

Take $n$ larger than the number of steps in the termination of $\mathfrak{C}\left[\operatorname{protect}_{\tau}\left(\operatorname{erase}\left(\mathrm{t}_{1}\right)\right)\right] \Downarrow$.

By Lemma 2, we have that $\emptyset \vdash \mathrm{t}_{1} \gtrsim_{n} \operatorname{erase}\left(\mathrm{t}_{1}\right): \tau$.

By Lemma 10, we then have (taking $m=n \geq n, p=$ precise and $\square=\gtrsim$ ) that

$$
\emptyset \vdash\langle\langle\mathfrak{C}\rangle\rangle_{\tau ; n}\left[\mathbf{t}_{1}\right] \gtrsim n \mathfrak{C}\left[\operatorname{protect}_{\tau}\left(\operatorname{erase}\left(\mathbf{t}_{1}\right)\right)\right]: \text { EmulDV } V_{\mathbf{n} ; \text { precise }} .
$$

Now by Lemma 1 , by $\mathfrak{C}\left[\operatorname{protect}_{\tau}\left(\operatorname{erase}\left(\mathrm{t}_{1}\right)\right)\right] \Downarrow$, and by the choice of $n$, we have that $\langle\langle\mathfrak{C}\rangle\rangle_{\tau ; n}\left[\mathrm{t}_{1}\right] \Downarrow$.

It now follows from $\emptyset \vdash \mathbf{t}_{1} \simeq_{c t x} \mathbf{t}_{2}: \tau$ and $\langle\langle\mathfrak{C}\rangle\rangle_{\tau ; n}\left[\mathbf{t}_{1}\right] \Downarrow$ that $\langle\langle\mathfrak{C}\rangle\rangle_{\tau ; \mathrm{n}}\left[\mathbf{t}_{2}\right] \Downarrow$.

Now take $n^{\prime}$ the number of steps in the termination of $\left\langle\left\langle\mathfrak{C}^{c}\right\rangle\right\rangle_{\tau ; n}\left[\mathbf{t}_{2}\right] \Downarrow$. We have from Lemma 2 that $\emptyset \vdash \mathrm{t}_{2} \lesssim_{n^{\prime}}$ erase $\left(\mathrm{t}_{2}\right): \tau$.

By Lemma 10, we then have (taking $m=n, n=n^{\prime}, p=$ imprecise and $\square=\lesssim$ ) that

$\emptyset \vdash\langle\langle\mathfrak{C}\rangle\rangle_{\tau ; \mathbf{n}}\left[\mathbf{t}_{\mathbf{2}}\right] \lesssim n^{\prime} \mathfrak{C}\left[\operatorname{protect}_{\tau}\left(\operatorname{erase}\left(\mathrm{t}_{2}\right)\right)\right]:$ EmulDV$_{\mathbf{n} ; \text { imprecise }}$

Now by Lemma 1 , by $\left\langle\left\langle\mathfrak{C}^{2}\right\rangle\right\rangle_{\tau ; n}\left[\mathrm{t}_{2}\right] \Downarrow$, and by the choice of $n^{\prime}$, we have that $\mathfrak{C}\left[\operatorname{protect}_{\tau}\left(\operatorname{erase}\left(\mathrm{t}_{2}\right)\right)\right] \Downarrow$ as required.

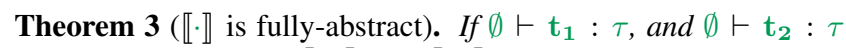
then $\mathrm{t}_{1} \simeq_{c t x} \mathrm{t}_{2} \Longleftrightarrow \llbracket \mathrm{t}_{1} \rrbracket \simeq_{c t x} \llbracket \mathrm{t}_{2} \rrbracket$.

Proof. Theorem 1 provides the $\Leftarrow$ direction while Theorem 2 provides the $\Rightarrow$ one.

Note that extending the above theorem to open terms would require the compiler to confine free variables to their type.

\section{Discussion and Future Work}

Our interest in fully-abstract compilation comes from a security perspective. We think that a fully-abstract compiler from realistic source languages to a form of assembly that is efficiently executable by processors has important security applications (combining trusted and untrusted code at the assembly level and compartmentalising applications). So far, it remains unclear precisely which security properties are preserved by fully abstract compilers, although it seems that at least important security properties like noninterference [Bowman and Ahmed, 2015] are. Unless targeting typed assembly language [Morrisett et al., 1999], a crucial step of a secure compiler is a form of secure type erasure. The contribution of this paper is mostly the proof technique that proves the type erasure step secure. We intend to reuse this proof technique in other settings.

There are a number of important problems that need to be solved in order to develop a realistic fully-abstract compiler. Several widely-implemented high-level language features present significant challenges: parametric polymorphism, (higher-order) references, exceptions etc. Generally, we believe that low-level assembly languages should be defined that are not only efficiently executable but also provide sufficient abstraction features to enable fully abstract compilation of such standard programming language features. For now, it remains an open question whether this is feasible. Let us zoom in on some of these features in more detail. A long-standing open problem is fully-abstract compilation of parametric polymorphism to a form of operational sealing primitives [Sumii and Pierce, 2007, Matthews and Ahmed, 2008, Neis et al., 2009]. More concretely, several researchers have developed interesting results about fully-abstract compilation from System F to $\lambda^{\text {seal }}$ (an untyped lambda calculus with sealing primitives), but a fully-abstract compiler in this setting has so far only been conjectured. We believe that the problem is quite related to the one tackled in this paper. Without providing details (for space reasons), an exact back-translation from $\lambda^{\text {seal }}$ to System F seems possible, but only if we assume a form of generally recursive type constructors of kind $* \rightarrow *$, which we cannot add to System $\mathrm{F}$ without causing other problems for the compilation. We conjecture an approximate backtranslation is what is needed to provide a fully-abstract compilation in this setting and we hope to confirm it in future work.

In other settings, it is not clear whether it is even possible to construct a fully-abstract compiler. For example, if we add typed, higher-order references to $\lambda^{\tau}$ and untyped references to $\lambda^{\mathrm{u}}$, it is not clear if a fully-abstract compiler can be devised. The problem is essentially to choose a representation for typed references and a way of manipulating them that reconciles a number of requirements: (1) trusted code reading from a reference always produces a type-correct value, (2) trusted code writing a type-correct value to a reference always works, (3) untrusted code should be able to read/write type-correct values from references, (4) dynamic type checks or wrappers may only be added where the context could also choose to fail for other reasons (i.e. not at the time of reading/writing a reference by trusted code), (5) efficiency: we do not want to check the contents of all references every time control is passed from trusted code to the context. Several obvious solutions do not work: representing references as objects with read and write methods violates requirement (4), just checking the contents of a reference when it is received from the context is not enough to guarantee (1) and (2). We intend to explore a solution based on trusted but abstract read/write/alloc methods (using sealing primitives as used for parametric polymorphism) but this remains speculation for the moment.

Another interesting problem when compiling to an assembly language is the enforcement of well-bracketed control flow. The question is essentially how to represent return pointers at the assembly level. Even if we prevent functions from accessing parts of the stack and only give them access to an opaque invokable return pointer, they still have ways to misuse them [Patrignani, 2015]. Imagine a trusted assembly function $f$ invoking an untrusted $g$. Additionally, assume that $g$ in turn re-invokes $f$ and $f$ simply reinvokes $g$ again. Now $g$ might attempt to invoke the wrong return pointer, returning on its first invocation without first returning on the second. Such an attack breaks the well-bracketedness of control flow that trusted code may rely on in languages without call/cc primitives [Dreyer et al., 2010]. Ahmed and Blume [2011] have demonstrated a solution for this problem which exploits parametric polymorphism to enforce the invocation of the correct continuation, and it is interesting to see if their work can be reused as an intermediate step on the way to assembly language.

On a technical level, we expect few problems for applying our technique of approximate back-translation to all of these settings. The Hur-Dreyer-inspired cross-language logical relations can be applied in diverse settings including ML and assembly and support references (through Kripke worlds), parametric polymorphism (through quantification over abstract type interpretations as relations) and well-bracketed control flow guarantees (through public/private transitions in the transition systems stored in the worlds). We have also shown in this paper that they can be easily modified to an asymmetric setting.

\section{Related Work}

Secure compilation through full-abstraction was pioneered by Abadi [1999] and successfully applied to many different settings [Patrignani et al., 2015, Fournet et al., 2013, Bowman and Ahmed, 2015, Ahmed and Blume, 2011, 2008, Tse and Zdancewic, 
2004, Shikuma and Igarashi, 2007, Abadi and Plotkin, 2012, Jagadeesan et al., 2011, Riecke, 1993, Ritter and Pitts, 1995, Mitchell, 1993, McCusker, 1996, Smith, 1998]. Recently, Gorla and Nestman [2014] have argued against the use of the mere existence of fully-abstract translations as a measure of language expressiveness, because very often fully abstract translations exist but are in some sense degenerate, uninteresting and/or unrealistic. Recently, Parrow [2014] has strengthened their case by showing that fully abstract translations almost always exist (under a basic condition on the cardinality of equivalence classes in source and target language). Their argument is valid, but does not apply to applications of fully abstract compilers for security, which is our motivation.

Some secure compilation works prove compiler full-abstraction using logical relations. Ahmed and Blume [2011, 2008] proved that typed closure conversion and CPS transformation are fully-abstract when compiling from System $\mathrm{F}$ and the simply-typed $\lambda$-calculus (respectively) to System F. Tse and Zdancewic [2004] started a line of work to compile the dependency core calculus of Abadi et al. [1999] (DCC) into System F, effectively proving that non interference can be encoded with parametricity. They achieve an analogous of fully-abstract compilation where contextual equivalence is replaced with non-interference. Due to an imprecision in their proof, the result of Tse and Zdancewic does not hold; Shikuma and Igarashi [2007] refined their result for a weaker form of DCC. A fully-abstract translation from DCC to System F was provided by Bowman and Ahmed [2015], and that is the closest work to what is presented here. While they prove an important result, we believe the formal machinery adopted by Bowman and Ahmed is heavier than the one presented here. Specifically, we do not need a new logical relation to prove well-foundedness of the back-translation. The secure compilation of DCC to System F is quite different from our setting, since our target language is untyped.

Many other secure compilation works prove full-abstraction by replacing target-level contextual equivalence with another equivalent equivalence (most times it is trace equivalence or bisimilarity) [Fournet et al., 2013, Abadi and Plotkin, 2012, Jagadeesan et al., 2011, Patrignani et al., 2015]. These works rely on additional results of the equivalence used for full-abstraction to hold, and this can complicate and lengthen proofs relying on this other technique. Earlier, McCusker [1996] has previously shown that proving full abstraction of a compiler can be simplified by limiting the backtranslation to contexts that are in a certain sense compact. This is related to our approximate back-translations, though not quite the same. A downside of McCusker's approach is that it does not always seem clear how to characterize the compact elements in a language.

As mentioned in Section 1, the presented proof technique borrows from recent results in compiler correctness [Hur and Dreyer, 2011, Benton and Hur, 2010, 2009]. These results build crosslanguage logical relation based on a common language specification in order to prove compiler correctness. Benton and Hur [2009] provided a correct compiler from a call-by-value $\lambda$-calculus as well as for System $F$ with recursion to to a SECD machine [Benton and Hur, 2010]. Hur and Dreyer [2011] devised a correct compiler between an idealised ML to assembly. The techniques devised in these works were further developed into Relational Transition Systems (RTS) in order to prove both vertically- and horizontallycomposable compiler correctness [Hur et al., 2012, Neis et al., 2015]. A different approach to cross-language relations could have been adopting a Matthews and Findler-style multi-language semantics, where source and target language are combined [Matthews and Findler, 2009]. For example, Perconti and Ahmed [2014] devised a two-step correct compiler for System F with existential and recursive types to typed assembly language using multi-language logical relations. As compiler full-abstraction does scale to multi-pass compilers (i.e., it is vertically composable), there was no necessity to use RTS nor multi-language systems.

Some elements of our proof technique are reminiscent of techniques from the field of denotational semantics. First, our family of types $\mathrm{UVal}_{n}$ can be seen as a kind of syntactical version of an iteratively constructed Scott model for the untyped lambda calculus [Scott, 1976]. In fact, in future work, we hope to extend this correspondence to languages with effects, where the effects would be encoded with a state-passing model. We note also that using a family of finite approximations (like our $\mathrm{UVal}_{n}$ types) to interpret a recursive type (like the type UVal discussed in the introduction) is quite standard in denotational semantics [MacQueen et al., 1984].

\section{Conclusion}

This paper presented a novel proof technique for proving compiler full-abstraction based on asymmetric, cross-language logical relations. The proof technique revolves around an approximate backtranslations from target terms (and contexts) to source terms (and contexts). The back-translation is approximate in the sense that the context generated by the back-translation may diverge when the target-level counterpart would not, but not vice versa. The proof technique is demonstrated for a compiler from a simply-typed $\lambda$ calculus without recursive types to the untyped $\lambda$-calculus; that compiler is proven to be fully-abstract. Although logical relations have been used for full-abstraction proofs, this is the first usage of cross-language logical relations for compiler full-abstraction targeting an untyped language. We believe the techniques developed in this paper scale to languages with more advanced functionalities and they can be used to prove compiler full-abstraction in richer settings.

\section{Acknowledgments}

Dominique Devriese holds a Postdoctoral mandate from the Research Foundation Flanders (FWO). Marco Patrignani held a Ph.D. fellowship from the Research Foundation Flanders (FWO) during the development of this work. This research is partially funded by project grants from the Research Fund KU Leuven, and from the Research Foundation Flanders (FWO).

\section{References}

M. Abadi. Protection in programming-language translations. In Secure Internet programming, pages 19-34. Springer-Verlag, 1999. ISBN 3-540-66130-1.

M. Abadi and G. D. Plotkin. On protection by layout randomization. ACM Transactions on Information and System Security, 15: 8:1-8:29, July 2012. ISSN 1094-9224. doi: 10.1145/2240276. 2240279.

M. Abadi, A. Banerjee, N. Heintze, and J. G. Riecke. A core calculus of dependency. In Principles of Programming Languages, pages 147-160. ACM, 1999. doi: 10.1145/292540.292555.

P. Agten, R. Strackx, B. Jacobs, and F. Piessens. Secure compilation to modern processors. In Computer Security Foundations, pages 171-185, 2012.

A. Ahmed and M. Blume. Typed closure conversion preserves observational equivalence. In International Conference on Functional Programming, pages 157-168. ACM, 2008. doi: 10.1145/ 1411204.1411227.

A. Ahmed and M. Blume. An equivalence-preserving CPS translation via multi-language semantics. In International Conference on Functional Programming, pages 431-444. ACM, 2011. doi: 10.1145/2034773.2034830. 
N. Benton and C.-K. Hur. Biorthogonality, step-indexing and compiler correctness. In International Conference on Functional Programming, volume 44, pages 97-108. ACM, 2009. doi: $10.1145 / 1596550.1596567$.

N. Benton and C.-K. Hur. Realizability and compositional compiler correctness for a polymorphic language. Technical report, MSR, 2010.

W. J. Bowman and A. Ahmed. Noninterference for free. In International Conference on Functional Programming. ACM, 2015.

P.-L. Curien. Definability and full abstraction. Electron. Notes Theor. Comput. Sci., 172:301-310, 2007. ISSN 1571-0661. doi: 10.1016/j.entcs.2007.02.011.

D. Devriese, M. Patrignani, and F. Piessens. Fully abstract compilation by approximate back-translation: Technical appendix. Technical Report CW 687, Dept. of Computer Science, KU Leuven, 2016.

D. Dreyer, G. Neis, and L. Birkedal. The impact of higher-order state and control effects on local relational reasoning. In International Conference on Functional Programming, pages 143-156, 2010. doi: $10.1145 / 1863543.1863566$.

C. Fournet, N. Swamy, J. Chen, P.-E. Dagand, P.-Y. Strub, and B. Livshits. Fully abstract compilation to JavaScript. In Principles of Programming Languages, pages 371-384. ACM, 2013. doi: $10.1145 / 2429069.2429114$.

D. Gorla and U. Nestman. Full abstraction for expressiveness: History, myths and facts. Math. Struct. Comp. Science, 2014.

C.-K. Hur and D. Dreyer. A Kripke logical relation between ML and assembly. In Principles of Programming Languages, pages 133-146. ACM, 2011. doi: 10.1145/1926385.1926402.

C.-K. Hur, D. Dreyer, G. Neis, and V. Vafeiadis. The marriage of bisimulations and Kripke logical relations. In Principles of Programming Languages, pages 59-72. ACM, 2012. doi: 10.1145/2103656.2103666.

R. Jagadeesan, C. Pitcher, J. Rathke, and J. Riely. Local memory via layout randomization. In Computer Security Foundations Symposium, pages 161-174. IEEE Computer Society, 2011. doi: 10.1109/CSF.2011.18.

A. Kennedy. Securing the .NET programming model. Theor. Comput. Sci., 364(3):311-317, Nov. 2006. ISSN 0304-3975. doi: $10.1016 /$ j.tcs.2006.08.014.

D. MacQueen, G. Plotkin, and R. Sethi. An ideal model for recursive polymorphic types. In Principles of Programming Languages, pages 165-174. ACM, 1984. doi: 10.1145/800017. 800528 .

J. Matthews and A. Ahmed. Parametric polymorphism through run-time sealing or, theorems for low, low prices! In Programming Languages and Systems, volume 4960 of LNCS, pages 16-31. Springer Berlin Heidelberg, 2008. doi: 10.1007/ 978-3-540-78739-6_2.

J. Matthews and R. B. Findler. Operational semantics for multilanguage programs. ACM Transactions on Programming Languages and Systems, 31:12:1-12:44, Apr. 2009. ISSN 01640925. doi: 10.1145/1498926.1498930.

G. McCusker. Full abstraction by translation. Advances in Theory and Formal Methods of Computing, 1996.

J. C. Mitchell. On abstraction and the expressive power of programming languages. Science of Computer Programming, 21(2):141
- 163, 1993. ISSN 0167-6423. doi: 10.1016/0167-6423(93) 90004-9.

G. Morrisett, K. Crary, N. Glew, D. Grossman, R. Samuels, F. Smith, D. Walker, S. Weirich, and S. Zdancewic. TALx86: A realistic typed assembly language. In Second Workshop on Compiler Support for System Software, pages 25-35, 1999.

G. Neis, D. Dreyer, and A. Rossberg. Non-parametric parametricity. In International Conference on Functional Programming, pages 135-148. ACM, 2009. doi: 10.1145/1596550.1596572.

G. Neis, C.-K. Hur, J.-O. Kaiser, C. McLaughlin, D. Dreyer, and V. Vafeiadis. Pilsner: A compositionally verified compiler for a higher-order imperative language. In International Conference on Functional Programming. ACM, 2015.

J. Parrow. General conditions for full abstraction. Math. Struct. Comp. Science, 2014.

M. Patrignani. The Tome of Secure Compilation: Fully Abstract Compilation to Protected Modules Architectures. PhD thesis, KU Leuven, Leuven, Belgium, May 2015.

M. Patrignani, P. Agten, R. Strackx, B. Jacobs, D. Clarke, and F. Piessens. Secure compilation to protected module architectures. ACM Trans. Program. Lang. Syst., 37(2):6:1-6:50, Apr. 2015. ISSN 0164-0925. doi: 10.1145/2699503.

J. T. Perconti and A. Ahmed. Verifying an open compiler using multi-language semantics. In ESOP, volume 8410 of Lecture Notes in Computer Science, pages 128-148, 2014.

B. C. Pierce. Types and programming languages. MIT press, 2002.

G. D. Plotkin. LCF considered as a programming language. Theoretical Computer Science, 5:223-255, 1977. doi: 10.1016/ 0304-3975(77)90044-5.

J. G. Riecke. Fully abstract translations between functional languages. Mathematical Structures in Computer Science, 3:387-415, 12 1993. ISSN 1469-8072. doi: 10.1017/ S0960129500000293.

E. Ritter and A. M. Pitts. A fully abstract translation between a İ̀z-calculus with reference types and standard ml. In M. DezaniCiancaglini and G. Plotkin, editors, Typed Lambda Calculi and Applications, volume 902 of LNCS, pages 397-413. Springer Berlin Heidelberg, 1995. ISBN 978-3-540-59048-4. doi: 10. 1007/BFb0014067.

D. Scott. Data types as lattices. SIAM Journal on Computing, 5(3): 522-587, 1976. doi: 10.1137/0205037.

N. Shikuma and A. Igarashi. Proving noninterference by a fully complete translation to the simply typed $\lambda$-calculus. In M. Okada and I. Satoh, editors, Advances in Computer Science ASIAN 2006. Secure Software and Related Issues, volume 4435 of LNCS, pages 301-315. Springer Berlin Heidelberg, 2007. doi: 10.1007/978-3-540-77505-8_24.

S. F. Smith. The coverage of operational semantics. In Higher Order Operational Techniques in Semantics, Publications of the Newton Institute, pages 307-346. Cambridge University Press, 1998.

E. Sumii and B. C. Pierce. A bisimulation for dynamic sealing. Theor. Comput. Sci., 375(1-3):169-192, Apr. 2007. ISSN 03043975. doi: $10.1016 /$ j.tcs.2006.12.032.

S. Tse and S. Zdancewic. Translating dependency into parametricity. In International Conference on Functional Programming, pages 115-125. ACM, 2004. doi: 10.1145/1016850.1016868. 